\title{
Structure of the image of (pseudo)-holomorphic discs with totally real boundary condition
}

\author{
DAEsung Kwon ${ }^{1}$ AND Yong-Geun $\mathrm{OH}^{2}$
}

In this paper, we prove that the image of a (pseudo)-holomorphic map from the unit disc with its boundary on a given totally real submanifold is represented as a finite union of the images of "simple" discs allowing multiplicity with boundaries on the same totally real submanifold. This in particular fills a technical gap in relation to the Fredholm and the intersection theory of Gromov's (pseudo)holomorphic discs, which has been present in the literature on the applications of pseudo-holomorphic discs.

With an appendix by Jean-Pierre Rosay

\section{Introduction.}

In recent papers [O3,4], the second author has studied the structure of $J$ holomorphic discs with totally real boundary condition, in relation to the Fredholm theory of $J$-holomorphic discs and its application to the problems of symplectic topology of Lagrangian submanifolds. We refer the readers to $[\mathrm{O} 3,4]$ for some motivation and importance of such a study. We also refer to $[\mathrm{C}]$ for the study of local structure of the image (near singularities) of classical analytic discs with totally real boundary conditions in several complex variables. This paper $[\mathrm{C}]$ in particular contains the examples that show how complex can be the local structure of boundary singularities of analytic discs with smooth, but not real analytic, totally real boundary conditions even in the classical context.

A general perception in the literature concerning the Gromov theory of $J$-holomorphic discs, misguided by the experience from the results on the $J$ holomorphic spheres, has been that as in the case of $J$-holomorphic spheres, any $J$-holomorphic disc should satisfy either

\footnotetext{
${ }^{1}$ Partially supported by GARC and BSRI

${ }^{2}$ Partially supported by the NSF Grant \# DMS-9504455
} 
(i)

(1.1) $d w(z) \neq 0$ and $w^{-1}(w(z))=\{z\}$ for some point $z \in D^{2}$ or

(ii) it should be a multiple covering of a J-holomorphic disc satisfying (1.1).

(See e.g. [P], [O2] and [L].) Just as the applications of $J$-holomorphic spheres to the structure of rational symplectic 4-manifolds [M2], to the Floer homology of symplectic manifolds [F], [HS] and to the theory of GromovWitten invariants and quantum cohomology [R], [RT], [MS] have illustrated, this structure theorem for the spheres has been an important ingredient especially when one studies intersection theory and compactification of the moduli space of pseudo-holomorphic spheres. It is natural to believe that the corresponding structure theorem for discs will bear similar importance in its applications to symplectic topology of Lagrangian submanifolds (see $[\mathrm{P}],[\mathrm{L}]$ and [O2]), and to the theory of fillings by (pseudo-)holomorphic discs (see e.g., [H], [Y2]). This structure theorem has not been carefully addressed in the literature. See [Y2] for some study of structure of the image of pseudo-holomorphic discs in symplectic 4-manifolds under the "one sidedness" condition, which had been introduced by [BG] in the classical context of several complex variables.

The second author pointed out in $[03,4]$ that the presumed structure theorem for discs does not have the same form as mentioned above for spheres, and is a theorem to formulate and prove, which should require a non-trivial proof. In [O4], he also made a first step to this goal by proving the following theorem, among other things, which can be considered as the $J$-holomorphic analogue to the more classical theorem in several complex variables. (See e.g., [St], [GS]).

Theorem 1.1 [Theorem I, O4]. Let $R \subset(M, J)$ be a totally real submanifold and $w:\left(D^{2}, \partial D^{2}\right) \rightarrow(M, R)$ be a non-constant J-holomorphic map such that it extends smoothly to the boundary and

$$
\text { "Int } D^{2} \cap w^{-1}(b A) \text { is connected and simply connected }
$$
(after adding a discrete set of points)"

Then we can write

$$
w=\widetilde{w} \circ b
$$

where $\widetilde{w}:\left(D^{2}, \partial D^{2}\right) \rightarrow(M, R)$ is a J-holomorphic disc with smooth extension to the boundary, which is injective away from a discrete set of points 
in the interior of $D^{2}$ and $b: D^{2} \rightarrow D^{2}$ is a finite Blaschke product. In other words, $b$ is of the form with $s \geq 1$

$$
b(z)=e^{i c} \prod_{k=1}^{s} \frac{z-\alpha_{k}}{1-\bar{\alpha}_{k} z}
$$

where $c$ is a real constant and $\alpha_{k}$ are complex constants with $\left|\alpha_{k}\right|<1$.

Following [St], [O4], we call the map like $\widetilde{w}$ a simple map. In the above theorem, the hypothesis (1.2) cannot be dropped as shown by the following example given in [Remark 3.3, O3]: Consider the holomorphic map

$$
w: \mathbb{H} \rightarrow \mathbb{C}, \quad w(z)=z^{k}
$$

where $\mathbb{H} \subset \mathbb{C}$ is the upper half-plane. Identify $\mathbb{C} \cup\{\infty\}=S^{2}$ and $\mathbb{H} \cup\{\infty\}=$ $D^{2} \subset \mathbb{C}$ and $\partial \mathbb{H}=\mathbb{R} \subset \mathbb{C}$. If $k$ is odd and at least 5 , this map does not satisfy (1.1) but cannot be written as a multiple covering of a simple disc. In this example, the set Int $D^{2} \backslash w^{-1}(b A)$ is not connected. See [Example 2.4, La] for the case when Int $D^{2} \backslash w^{-1}(b A)$ is connected but not simply connected even after adding a discrete set of points.

These examples show that structure of the images of $J$-holomorphic discs is more complex than that of spheres in general. What makes things more complex for the case of discs, unless we impose the condition (1.2), is that unlike from the case of spheres, the multiplicity of the image point will not be constant but vary as the examples above show. Certainly any map of the form in (1.3) has constant multiplicity. In fact, as far as we know, most of the results in the classical theory of analytic discs in several complex variables escape this unpleasant phenomenon because they usually consider proper holomorphic maps of the type

$$
f: \Delta \rightarrow \mathbb{C}^{N} \backslash \Gamma, \quad \Delta=\operatorname{Int} D^{2}
$$

where $\Gamma \subset \mathbb{C}^{N}$ is a compact subset of $\mathbb{C}^{N}$ (e.g. $\Gamma=$ totally real submanifolds). In other words, these cases do not allow the boundary image to pierce through that of the interior. In respect to this, the main structure theorem below may be new even in the classical context of analytic discs in several complex variables.

The above mentioned non-constantness of the multiplicity is due to the "piercing" of the boundary into the interior when we do not assume (1.2). It has not been a priori clear what would be the correct formulation of the structure theorem in general, which question was left open in [04]. However 
based on the results from [M1,3,4] and [O4], it is not difficult to show that the set $\Delta \backslash w^{-1}(b A)$ is a finite disjoint union of open domains in $\Delta \subset \mathbb{C}$. (See Corollary 4.3 below.) Each of these domains, after possibly adding a discrete set of points in the interior will become an open domain in $\Delta$ with genus $g$ possibly bigger than zero. Then the main question to ask is whether domains with genus $g \geq 1$ will really occur in this union. We denote

$$
\Delta \backslash w^{-1}(b A)=\bigcup_{j=1}^{\ell} E_{j}
$$

and by $\widetilde{E}_{j}$ the domain obtained by adding a discrete set of points in $E_{j}$. (See Section 4 for more precise definition of $\widetilde{E}_{j}$.)

It turns out that $\widetilde{E}_{j}$ are always simply connected when $(M, J)$ does not allow a $J$-holomorphic sphere but high genus components can occur if $(M, J)$ admits a $J$-holomorphic sphere (see Example and Corollary 4.11 in Section 4). This gives rise to the following interesting result in $\mathbb{C}^{n}$.

Corollary. If $J$ is any almost complex structure tame to the standard symplectic structure on $\mathbb{C}^{n}$ (e.g., the standard complex structure on $\mathbb{C}^{n}$ ), $\widetilde{E}_{j}$ defined as above are all simply connected for any J-holomorphic disc $w:\left(D^{2}, \partial D^{2}\right) \rightarrow\left(\mathbb{C}^{n}, R\right)$.

However even when $(M, J)$ allows a $J$-holomorphic sphere, we can still prove the following theorem which proves that at least the image of $\widetilde{E}_{j}$ under $w$ is the same as that of a map from $\left(D^{2}, \partial D^{2}\right) \rightarrow(M, R)$ (see Lemma 4.6 and its proof for the details).

Theorem I [Theorem 4.4]. Let $\left\{E_{j}\right\}_{j=1}^{\ell}$ be the connected components in the union (1.5) and $\widetilde{E}_{j}$ be as above. Then we can further decompose, if necessary, each domain $\widetilde{E}_{j}$ into the finite union $\cup_{i} \widetilde{E}_{j}^{i}$ so that $\left.w\right|_{\widetilde{E}_{j}^{i}}$ can be reduced to a map $w_{j}^{i}:\left(D^{2}, \partial D^{2}\right) \rightarrow(M, R)$ with $\operatorname{Im} w_{j}^{i}=\left.\operatorname{Im} w\right|_{\widetilde{E}_{j}^{i}}$, and

$$
\left[\left.w\right|_{\widetilde{E}_{j}}\right]=\sum_{i}\left[w_{j}^{i}\right] \quad \text { in } H_{2}(M, R ; \mathbb{Z})
$$

(See Corollary 4.11 for more detailed structure of the image of $w$ when some $\widetilde{E}_{j}$ is not simply connected.)

Since $w_{j}^{i}$ 's satisfy (1.2), combining Theorem 1.1 and Theorem I, we obtain our main structure theorem. 
Theorem II (Main Structure Theorem). The image of a J-holomorphic disc with totally real boundary condition can be represented as a finite union of simple J-holomorphic discs (with multiplicity) with the same totally real boundary condition. Furthermore this representation preserves the homotopy class in $\pi_{2}(M, R)$.

This theorem completes formulation and proof of the structure theorem that has been used in the literature. Once we have this structure theorem, one can then safely repeat the same kind of arguments as those for the sphere case in the generic transversality result, the structure of Gromov compactification of the moduli space of J-holomorphic discs and intersection theory of $J$-holomorphic discs that have been needed in the applications (e.g. in $[\mathrm{P}],[\mathrm{L}]$ and $[\mathrm{O} 2])$.

The proof of Theorem I turns out to involve quite complicated combinatorial arguments, which are originally due to the first author, based on the local structure of piercing by the boundary through the interior image. The essential tool for the analysis of this piercing phenomenon is Proposition 3.5. This is a unique continuation principle of the image along the boundary, which has some independent interest in its own right. Unlike the case of interior, the unique continuation fails in general due to "branching off" and so requires certain restrictions in its application as imposed in Proposition 3.5. We include several figures in the course of the proof, which we hope helps readers grasp main arguments in the proof.

We next study a finer structure of the "net" $w^{-1}(b A)$ in $\Delta$ and the image of $w:\left(D^{2}, \partial D^{2}\right) \rightarrow(M, R)$.

Let $\phi_{j}^{i}: \Delta \rightarrow \widetilde{E}_{j}^{i}$ (identified with a disc as in (4.16)) be a Riemann map and denote

$$
w_{j}^{i}=\left.w\right|_{\widetilde{E}_{j}^{i}} \circ \phi_{j}^{i}: \Delta \rightarrow M .
$$

Since $\left.w\right|_{\widetilde{E}_{j}^{i}}$ is continuous up to the boundary and so is the Riemann map $\phi_{j}^{i}: \Delta \rightarrow \widetilde{E}_{j}^{i}, w_{j}^{i}$ is continuous up to boundary with $w_{j}^{i}\left(\partial D^{2}\right) \subset R$. By the standard boundary regularity theorem, $w_{j}^{i}$ is smooth up to boundary. And it follows from the definition of $\widetilde{E}_{j}^{i}$ that $w_{j}^{i}$ allows a factorization like (1.3). We denote the corresponding simple map by $\widetilde{w}_{j}^{i}$ and the Blaschke product by $b_{j}^{i}$, i.e.,

$$
w_{j}^{i}=\widetilde{w}_{j}^{i} \circ b_{j}^{i} .
$$

We first quote the following theorem proven in [O4], which tells the structure of the image of simple maps. 
Theorem 1.2 [Theorem II, O4]. Let $\widetilde{w}:\left(D^{2}, \partial D^{2}\right) \rightarrow(M, R)$ be a simple J-holomorphic map. Then the following alternative holds:

(i) there is a point $z \in \partial D^{2}$ such that

$$
\widetilde{w}^{-1}(\widetilde{w}(z)) \cap \partial D^{2}=\{z\} \quad \text { and } \quad d \widetilde{w}(z) \neq 0 .
$$

(ii) the multiplicity of $\kappa: b A \backslash X \rightarrow \mathbb{Z}_{+}$is two except at a discrete set of points where $X$ is the set of critical values. Furthermore, the image $A:=$ Image $\widetilde{w}$ becomes a smooth immersed branched Riemann surface.

In particular, If $(M, J)$ does not carry any closed $J$-holomorphic Riemann surface (e.g. like $\mathbb{C}^{n}$ ), then only the alternative (i) must hold.

Definition 1.3. We call a simple map satisfying (i) type I and one satisfying (ii) type II.

Examples of type I and II are the maps in (1.4) for $k=1$ and $k=2$, respectively.

A priori, both types of the simple map could occur as $\widetilde{w}_{j}^{i}$ 's in the decomposition in Theorem II. Due to Theorem 1.2, we may assume that the number of components in the decomposition is greater than 1. It is rather surprising that in the decomposition in Theorem II, only one of the two types can occur.

Theorem III. Let $\cup_{j=1}^{k} \widetilde{E}_{j}^{i}$ be the decomposition in Theorem II, and $w_{j}^{i}, \widetilde{w}_{j}^{i}$ and $b_{j}^{i}$ defined as above, Then we have the alternative:

(i) all $\widetilde{w}_{j}^{i}$ 's are of type $I$

(ii) all $\widetilde{w}_{j}^{i}$ 's are of type II: In this case, the images of all $\widetilde{w}_{j}^{i}$ 's coincide as a set. Furthermore, in this case, the multiplicity of $\kappa: A \rightarrow \mathbb{Z}_{+}$is constant(on the open dense set of $A \backslash X$ where $X$ is the set of critical values).

If $J$ is integrable near $R$ and $R$ is real-analytic, we can strengthen (i) to

(i') all $\widetilde{w}_{j}^{i}$ 's are of type I and the union of the images of any two consecutive $\widetilde{w}_{j}^{i}, \widetilde{w}_{k}^{\ell}$ such that $j \neq k$, forms a closed branched Riemann surface.

Examples of the cases (i) and (ii) are provided by maps in (1.4) with $k$ odd and with $k$ even, respectively. 
As the example in the appendix shows, the result $\left(i^{\prime}\right)$ fails to hold if we just assume that $R$ is smooth (but not real analytic). See Proposition 6.6 where both integrability of $J$ and real-analyticity of $R$ are essential to apply the reflection principle.

Using Theorem III $\left(i^{\prime}\right)$, one can prove a more precise structure theorem than Theorem II for the case of integrable $J$ near $R$ and real-analytic $R$. We refer to Corollary 6.3 below for this. In fact in this case, the whole proof of Theorem I can be considerably simplified using Proposition 6.6. It rules out the branching phenomenon along the boundary in the real analytic case which complicates the image of general smooth cases as the map $h_{ \pm}$ illustrate in Rosay's example in the appendix.

Note that for the map $w:\left(D^{2}, \partial D^{2}\right) \rightarrow\left(\mathbb{C}^{n}, R\right)$ with respect to an almost complex structure $J$ tamed with the standard symplectic structure, the alternative (ii) was ruled out in Proposition 5.1. Furthermore from Theorem III $\left(i^{\prime}\right)$, it also follows that there cannot be more than one connected component in the above decomposition, provided $J$ is integrable near $R$ and $R$ is real-analytic. In particular, noting that $\mathbb{C}^{n}$ does not allow any closed holomorphic Riemann surface, this immediately implies the following result, which seems to be a new result even in several complex variables.

Corollary 6.3. Consider $\mathbb{C}^{n}$ with its standard complex structure $J_{0}$ and let $R$ be a real-analytic totally real submanifold in $\mathbb{C}^{n}$. Then for any holomorphic disc $w:\left(D^{2}, \partial D^{2}\right) \rightarrow\left(\mathbb{C}^{n}, R\right), \Delta \backslash w^{-1}(b A)$ is connected and simply connected (after adding a discrete set of points) and so $w$ allows the factorization (1.3).

After the first version of this paper was completed, responding to the question asked by the second author, J. -P. Rosay has constructed a counter example to Corollary 6.3 for a smooth $R$, which is included in Appendix 1. This example shows that the real-analyticity is essential in Corollary 6.3. And while this paper was in submission, Lazzarini sent us his preprint [La] that contains a result which is weaker than ours. But we learned from his paper [La] that there is some error in the statement of [Theorem I, O4] in that the second author overlooked the possibility that $\operatorname{Int} D^{2} \backslash w^{-1}(b A)$ is connected but not simply connected (after adding a discrete set of points).

The second author would like to thank Jean-Pierre Rosay for his contribution to the appendix. We would also like to thank the unknown referee for pointing out some error in our formulation of Theorem I in the previous version, providing the example in Section 4 and raising the question of the homology class which leads us to writing Appendix 3, and Lazzarini for 
sending his preprint to us.

\section{Preliminaries.}

In this section, we organize various theorems on the local structure of $J$ holomorphic discs which we borrow mainly from [M1], [FHS] and [O4].

Let $(M, J)$ be an almost complex manifold and let $R \subset M$ be a (maximally) totally real submanifold with respect to $J$, i.e.,

$$
\operatorname{dim} R=\frac{1}{2} \operatorname{dim} M \text { and } T_{x} R \pitchfork J T_{x} R \text { for all } x \in R .
$$

We denote by $D^{2} \subset \mathbb{C}$ the closed unit disc and $\Delta=D^{2} \backslash \partial D^{2}$ the open unit disc. A smooth map

$$
w:\left(D^{2}, \partial D^{2}\right) \rightarrow(M, R)
$$

is called a $J$-holomorphic disc with the boundary condition $R$ if $v$ satisfies

$$
\left\{\begin{array}{l}
J \circ d w=d w \circ j \\
w\left(\partial D^{2}\right) \subset R
\end{array}\right.
$$

where $j$ is the standard complex structure on $D^{2} \subset \mathbb{C}$. We also denote by $B_{\epsilon}(0)$ the open disc centered at 0 with radius $\epsilon>0$ and by $D_{\epsilon}(0)$ the semi-disc centered at 0 with radius $\epsilon>0$ and with boundary

$$
\partial D_{\epsilon}(0)=(-\epsilon, \epsilon) \subset \mathbb{R} \subset \mathbb{C}
$$

Following the notations from [O4], we denote

$$
\begin{aligned}
A & =\text { Image of }\left.w\right|_{D^{2}} \\
b A & =\text { Image of }\left.w\right|_{\partial D^{2}}
\end{aligned}
$$

and introduce the multiplicity function $\kappa: A \rightarrow \mathbb{Z}_{+}$.

Definition 2.1. We define the integer valued function $\kappa: A \rightarrow \mathbb{Z}_{+}$by

$$
\kappa(a)=\#\left(w^{-1}(a)\right), \quad a \in A
$$

and call it the multiplicity of $a$.

We now quote several results on the local structure of $J$-holomorphic discs (with boundary) from [FHS] or [MS], and [O4]. 
Lemma 2.2 [Corollary 2.3, FHS; Corollary 2.2, O4]. Let $(M, J)$ be an almost complex manifold and $R \subset M$ be a totally real submanifold with respect to $J$. Let $w:\left(D_{\epsilon}(0), \partial D_{\epsilon}(0)\right) \rightarrow(M, R)$ be a smooth solution of

$$
\left\{\begin{array}{l}
\frac{\partial w}{\partial x}+J(w) \frac{\partial w}{\partial y}=0 \\
w(x, 0) \in R \quad \text { for } x \in(-\epsilon, \epsilon)=\partial D_{\epsilon}(0) .
\end{array}\right.
$$

Then there exists $\delta>0$ such that $d w(z) \neq 0$ for all $z$ with $0<|z|<\delta$. The corresponding result also holds for J-holomorphic maps $u: B_{\epsilon}(0) \rightarrow M$.

Lemma 2.2 implies that the set of critical points of a non-constant $J$ holomorphic disc is discrete. In the following two lemmas, $E$ denotes either the disc or the semi-disc.

Lemma 2.3 [Proposition 3.1, FHS; Corollary 2.3, O4]. Let (M, $J)$ and $R$ as above and let $w_{1}, w_{2}: E_{\epsilon} \rightarrow \mathbb{C}^{n}$ be non-constant smooth solutions of (2.2). If the $\infty$-jets of $w_{1}$ and $w_{2}$ coincide with 0 , then

$$
w_{1} \equiv w_{2}
$$

Lemma 2.4 [Lemma 2.2.2, MS]. Let $w: E \rightarrow(M, J)$ be J-holomorphic with $d w(0) \neq 0$. Then there is a local chart $\alpha: U \rightarrow \mathbb{C}^{n}$ near $w(0)$ such that

$$
\begin{aligned}
& \alpha \circ w(z)=(z, 0, \ldots, 0) \\
& d \alpha(w(z)) \circ J(w(z))=i d \alpha(u(z))
\end{aligned}
$$

for $z \in E \cap w^{-1}(U)$.

Combining Lemma 2.3 and 2.4, one can deduce the following useful results. (See [Lemma 2.2.3, MS] or [Corollary 2.4, O4] for the proof, respectively.)

Lemma 2.5 [Lemma 2.2.3, MS]. Let $u, v: B_{\epsilon}(0) \rightarrow(M, J)$ be nonconstant $J$-holomorphic maps with

$$
u(0)=v(0), \quad d u(0) \neq 0 .
$$

Assume that there are sequences $z_{\mu}, \xi_{\mu} \in B_{\epsilon}(0)$ such that

$$
u\left(z_{\mu}\right)=v\left(\xi_{\mu}\right), \quad \lim _{\mu \rightarrow \infty} z_{\mu}=\lim _{\mu \rightarrow \infty} \xi_{\mu}=0, \quad z_{\mu} \neq 0 \neq \xi_{\mu} .
$$


Then there is a holomorphic map $\phi: B_{\delta}(0) \rightarrow B_{\epsilon}(0)$ with $0<\delta<\epsilon, \phi(0)=0$ and

$$
v=u \circ \phi
$$

on $B_{\delta}(0)$.

However we cannot expect the obvious analogoue of this for the semi-disc case. One reason for this is that the images can be in "different" sides of the totally real submanifold and another is that they can also branch off as Rosay's example in Appendix illustrates (see Remark 6.4 and Proposition $6.6)$.

For the semi-disc case, we could expect only the following. The proof used for Lemma 2.5 cannot be applied to the boundary point because general $J$-holomorphic disc cannot be extended across its boundary as a smooth $J$. holomorphic map, unless $J$ in integrable and $R$ is real analytic near the point. We refer readers to the proof in [O4] for this point.

Lemma 2.6. [Corollary 2.4, O4]. Let $w_{i}:\left(D_{\epsilon_{i}}(0), \partial D_{\epsilon_{i}}\right) \rightarrow(M, R)$ be J-holomorphic with

$$
w_{1}(0)=w_{2}(0), \quad d w_{1}(0) \neq 0
$$

Assume that

$$
\left.\operatorname{Im} w_{1}\right|_{\partial D_{\epsilon_{1}}(0)}=\left.\operatorname{Im} w_{2}\right|_{\partial D_{\epsilon_{2}}(0)} .
$$

Then the following alternative holds:

(i) $\operatorname{Im} w_{1}$ and $\operatorname{Im} w_{2}$ overlap on some open neighborhoods of 0 or

(ii) $d w_{2}(0) \neq 0$ and Im $w_{1} \cup \operatorname{Im} w_{2}$ smoothly matches up along the boundary $\partial D_{\epsilon}(0)$ near 0.

One special case in Lemma 2.6 is worthwhile to separately mention and will be very useful for later discussions: Suppose that for $i=1,2, w_{i}$ : $\left(D_{\epsilon_{i}}(0), \partial D_{\epsilon_{i}}(0)\right) \rightarrow(M, R)$ be $J$-holomorphic with

$$
w_{1}(0)=w_{2}(0), \quad d w_{1}(0) \neq 0 \neq d w_{2}(0)
$$

such that there are some neighborhoods $U_{1}$ and $U_{2}$ of 0 in $D_{\epsilon}(0)$ respectively and

$$
\left.\operatorname{Im} w_{1}\right|_{\partial D_{\epsilon_{1}}(0) \cap U_{1}}=\left.\operatorname{Im} w_{2}\right|_{\partial D_{\epsilon_{2}}(0) \cap U_{2}} .
$$


We assume that $w_{i}: U_{i} \rightarrow M$ are one-one, which can be always achieved by choosing smaller $U_{i}$ 's if necessary. Denote

$$
\begin{aligned}
& \partial D_{\epsilon_{1}}(0) \cap U_{1}=\left(-\delta_{1}^{1}, \delta_{2}^{1}\right)=: \Lambda_{1} \\
& \partial D_{\epsilon_{2}}(0) \cap U_{2}=\left(-\delta_{1}^{2}, \delta_{2}^{2}\right)=: \Lambda_{2}
\end{aligned}
$$

for some $\delta_{j}^{i}>0$. Now consider the diffeomorphism

$$
\left.\left(\left.w_{2}\right|_{\Lambda_{2}}\right)^{-1} \circ w_{1}\right|_{\Lambda_{1}}: \Lambda_{1} \rightarrow \Lambda_{2}
$$

We provide the boundary orientation to $\Lambda_{i} \subset \partial D_{\epsilon_{i}}(0)$ induced from the complex orientation in $D_{\epsilon_{i}}(0) \subset \mathbb{C}$.

Corollary 2.7. Let $w_{i}$ 's, $\Lambda_{i}$ 's as above. Then the following alternative holds:

(i) if the diffeomorphism (2.3) preserves the orientation, then Lemma 2.6 (i) must hold.

(ii) if the diffeomorphism (2.3) reverses the orientation, then Lemma 2.6 (ii) must hold.

\section{Local structure of piercing.}

As we mentioned in the introduction, it is essential to analyze how $b A$ pierces through $A$ for the purpose of understanding structure of the images of $J$ holomorphic discs. We study this piercing by analyzing the "net" on $\Delta$ provided by $w^{-1}(b A) \cap \Delta$. In this section, we derive several results concerning the local structure of this piercing based on the results from Section 2. We will always assume that all (local) $J$-holomorphic maps appearing below have a finite number of critical points, and a discrete set of isolated selfintersections points (away from the critical values). For example, all the results in this section will apply to the maps obtained by restricting the domain of any given $J$-holomorphic map $w:\left(D^{2}, \partial D^{2}\right) \rightarrow(M, R)$.

The following notion of the collar neighborhood turns out to be useful for this purpose.

Definition 3.1. For a point $z \in D^{2}$, we say that $U \subset D^{2}$ is a collar neighborhood of $z$ (or $\lambda$ ) if $\partial U=\lambda$ is an embedded piecewise smooth arc containing $z$. 
The following lemma tells us that if the boundary of $J$-holomorphic disc is contained in the interior image of another such disc near a regular point of it, then images of the two discs should overlap in a collar neighborhood of the boundary of the first disc. Because of the branching phenomenon as we mentioned before, we cannot expect this if we replace "the interior image" by the boundary image of the second disc.

Lemma 3.2. Let $u:\left(D_{\epsilon}(0), \partial D_{\epsilon}\right) \rightarrow(M, R)$ and $v: B_{\epsilon}(0) \rightarrow M$ be $J$ holomorphic. Let $\left\{z_{\mu}\right\} \subset \partial D_{\epsilon}^{2}$ and $\left\{w_{\mu}\right\} \subset B_{\epsilon}^{2}$ be sequences with

$$
\begin{aligned}
u\left(z_{\mu}\right)=v\left(w_{\mu}\right) & \text { and } \\
\lim _{\mu \rightarrow \infty} z_{\mu}=0, z_{\mu} \neq 0, & \lim _{\mu \rightarrow \infty} w_{\mu}=0, w_{\mu} \neq 0 .
\end{aligned}
$$

Suppose in addition that $d v(0) \neq 0$. Then there exists a collar neighborhood $U \subset D_{\epsilon}(0)$ of $z$ with $\lambda=\partial U$, an arc contained in $\partial D^{2}$ such that

$$
u(U) \subset v\left(B_{\epsilon}(0)\right) .
$$

Proof. Without loss of any generality, we may assume that $(M, R)=$ $\left(\mathbb{C}^{n}, \mathbb{R}^{n}\right)$ and we are given two $J$-holomorphic maps

$$
u: D_{\epsilon}(0) \rightarrow \mathbb{C}^{n}, \quad v: B_{\epsilon}(0) \rightarrow \mathbb{C}^{n}
$$

such that

$$
u\left(z_{\mu}\right)=v\left(w_{\mu}\right), \quad \lim _{\mu \rightarrow \infty} z_{\mu}=0=\lim _{\mu \rightarrow \infty} w_{\mu},
$$

by restricting the maps to neighborhoods of 0 respectively. By Lemma 2.4, we may further assume

$$
v(z)=(z, 0, \ldots, 0), \quad J(z, 0, \ldots, 0)=i
$$

for $z \in B_{\epsilon}(0)$ and

$$
u(z)=(a(z), b(z)), a(z) \in \mathbb{C}, b(z) \in \mathbb{C}^{n-1}
$$

for $z \in D_{\epsilon}(0)$ such that $\left.u\left(\partial D_{\epsilon}(0)\right) \subset \mathbb{R}^{n}\right)$ and $b\left(z_{\mu}\right)=0$.

If $\infty$-jet of $b(z)$ at 0 does not vanish, then there is a non-negative integer: $\ell$ such that $b(z)=O\left(|z|^{\ell}\right)$ and $b(z) \neq O\left(|z|^{\ell+1}\right)$ and hence $J(u(z))=i+$ $O\left(|z|^{\ell}\right)$. Consider the Taylor expansion up to order $\ell-1$ of the equation

$$
\frac{\partial u}{\partial x}+J(u(z)) \frac{\partial u}{\partial y}=0
$$


on $D_{\epsilon}(0)$. Then we have

$$
\frac{\partial u_{\ell}}{\partial x}+i \frac{\partial u_{\ell}}{\partial y}=0, \quad \text { for } z \in D_{\epsilon}(0)
$$

where $u_{\ell}$ is the Taylor expansion of $u$ up to order $\ell$. So, there exists a nonzero $a$ such that

$$
b(z)=a z^{\ell}+O\left(|z|^{\ell+1}\right) \quad \text { for } z \in D_{\epsilon}(0)
$$

and hence $b(z) \neq 0$ in some open neighborhood of 0 . This then implies that the images of $v$ and $u$ do not intersect away from 0 in the neighborhood, which contradicts the assumption in the lemma. Therefore $\infty$-jet of $b(z)$ at 0 must vanish.

Since $J(z, 0, \ldots, 0) \equiv i$ for $z \in B_{\epsilon}(0), b(z)$ satisfies the equation

$$
\begin{aligned}
0 & =\frac{\partial b}{\partial x}+p r_{2}\left(J(a, b) \frac{\partial u}{\partial y}\right) \\
& =\frac{\partial b}{\partial x}+p r_{2}\left(\left(i+\int_{0}^{1} D_{b}(J(a, b)) d \tau \cdot v_{2}\right) \frac{\partial u}{\partial y}\right) \\
& =\frac{\partial b}{\partial x}+i \frac{\partial b}{\partial y}+(A b)(z)
\end{aligned}
$$

in a neighborhood of $\partial D_{\epsilon}(0)$ in $D_{\epsilon}(0)$ where $p r_{2}: \mathbb{C}^{n} \rightarrow \mathbb{C}^{n-1}$ is the projection and the operator $A$ is defined by

$$
(A \xi)(z):=\operatorname{pr}_{2}\left(\int_{0}^{1} D_{b}(J(a, \tau b) d \tau) \cdot \xi \frac{\partial u}{\partial y}\right)(z) .
$$

Since $b(x, 0) \in \mathbb{R}^{n-1}$ and the $\infty$-jet of $b$ at 0 vanishes, By Lemma 2.3,

$$
b(z) \equiv 0 .
$$

This finishes the proof.

An immediate corollary, when combined with Lemma 4.1 and 4.2 in the next section (see especially the remark after Lemma 4.2), is

Corollary 3.3. Let $w:\left(D^{2}, \partial D^{2}\right) \rightarrow(M, R)$ be a J-holomorphic disc with $d w\left(z^{\prime}\right) \neq 0$ for some $z^{\prime} \in w^{-1}(w(z)) \cap \Delta$ where $z \in \partial D^{2}$. Suppose that $\lambda_{0} \subset \partial D^{2}$ is an arc with $z$ as one end point and that there exists a collar neighborhood $V$ of $\lambda_{0}$ such that

$$
w(V) \subset w(\Delta)
$$


Then there exists a collar neighborhood $U$ of an open arc $\lambda=\partial U$ containing $z$ in its interior with

$$
w(U) \subset w(\Delta) .
$$

This corollary shows that the following branching picture of the images cannot occur.

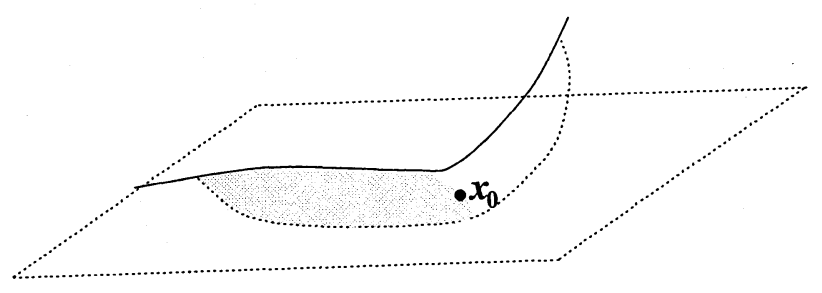

Figure 1.

The following objects appear often in later discussions and so we assign a special name to them.

Definition 3.4. We call $k$-armed asterisk the union of $k$ embedded arcs $\lambda_{1}, \ldots, \lambda_{k}$ in the plane that share one end point $z$ from each as drawn in Figure 2. We call semi-asterisk an asterisk $\cup_{j=1}^{k} \lambda_{j} \subset D_{\epsilon}(z)$ with

$$
\lambda_{1} \cup \lambda_{k} \subset \partial D_{\epsilon}(z)
$$

as drawn in Figure 2. We call each $\lambda_{j}$ in the asterisk an arm and the shared end point the center of the asterisk. We always order the arms either clockwise or counterclockwise in the plane. 


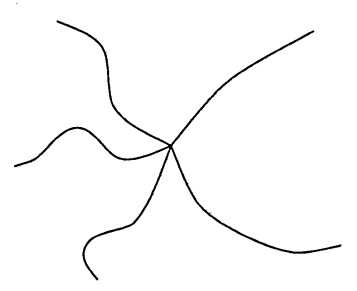

5-armed asterisk

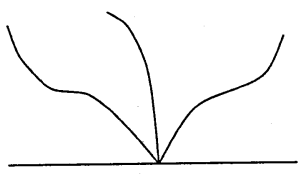

5-armed semi-asterisk

Figure 2.

The following proposition is the crucial tool in the proof of the main theorem. Roughly speaking, it shows that as long as the boundary of one disc does not intersect with the interior of the other, "overlap" of the images of two discs uniquely propagates along the boundary of discs. Recall that it is a well-known and an immediate consequence of Lemma 2.3 that overlap of the interior images of the two discs uniquely propagates.

Proposition 3.5. (Unique continuation of the image).

Let $w_{i}:\left(D_{\epsilon_{i}}(0), \partial D_{\epsilon_{i}}(0)\right) \rightarrow(M, R)$ for $i=1,2$ be two J-holomorphic maps satisfying the following properties:

(i) they can be smoothly extended to a neighborhood $D_{\epsilon_{i}^{\prime}}$ with $\epsilon_{i}^{\prime}>\epsilon_{i}$ of $\overline{D_{\epsilon_{i}}(0)}$ in $\mathbb{H} \subset \mathbb{C}$.

(ii) $w_{1}(0)=w_{2}(0)$

(iii) $d w_{i}(z) \neq 0, w_{i}(z) \neq w_{i}(0)$ for any $z$ with $0<|z|<\epsilon_{i}^{\prime}$ respectively for $i=1,2$.

(iv) Int $D_{\epsilon_{i}}(0) \cap w_{i}^{-1}\left(w_{j}\left(\partial D_{\epsilon_{j}^{\prime}}(0)\right)\right)$ is discrete in $\overline{D_{\epsilon_{i}}} \backslash\{0\}$, for all $i, j=$ 1,2 .

(v) there exists some $d>0$ such that

$$
\operatorname{dist}\left(p, w_{i}\left(\overline{D_{\epsilon_{i}}} \backslash D_{\epsilon_{i}}\right)\right)>d>0
$$


where $p=w_{1}(0)=w_{2}(0)$.

If there exist collar neighborhoods $U_{i}$ of $\left(-\epsilon_{i}, 0\right)$ in $D_{\epsilon_{i}}(0)$ with $\partial U_{i}=$ $\left(-\epsilon_{i}, 0\right)$ for $i=1,2$ and with $w_{1}\left(U_{1}\right)=w_{2}\left(U_{2}\right), w_{1}\left(\partial U_{1}\right)=w_{2}\left(\partial U_{2}\right)$, then we can find collar neighborhoods $V_{i}$ of $\left(-\epsilon_{i}, \delta_{i}\right)$ such that $\delta_{i}>0$ and

$$
w_{1}\left(V_{1}\right)=w_{2}\left(V_{2}\right)
$$
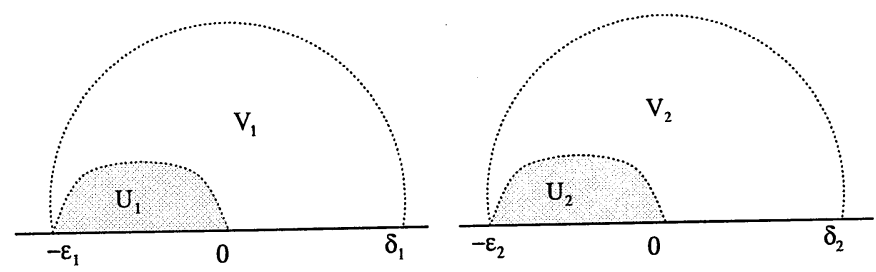

Figure 3.

Remark 3.6. (i) The hypothesis (v) can be always obtained by choosing $\epsilon_{i}^{\prime} s$ smaller if necessary.

(ii) This proposition fails to be true if we drop the hypothesis (iv), i.e., allow the image of the boundary to pierce through that of the interior. For example, consider the two maps

$$
w_{1}(z)=z, \quad w_{2}(z)=z^{2}:\left(D_{\epsilon}(0), \partial D_{\epsilon}(0)\right) \rightarrow(\mathbb{C}, \mathbb{R})
$$

and start with the intervals $\left(0, \epsilon_{i}\right)$ for appropriate $0<\epsilon_{i}<\epsilon$ for $i=1,2$.

(iii) This proposition rules out the following branching phenomenon under the hypotheses in the proposition, which otherwise could occur. Compare with Figure 1. 


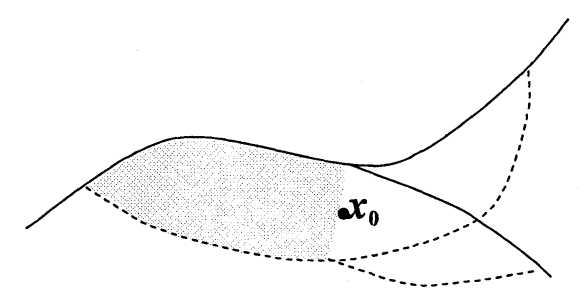

Figure 4.

To prove this proposition, we start with two lemmas. The first lemma rules out folding of the image (See Figure 5). The second one is a continuation lemma of the collar neighborhood.

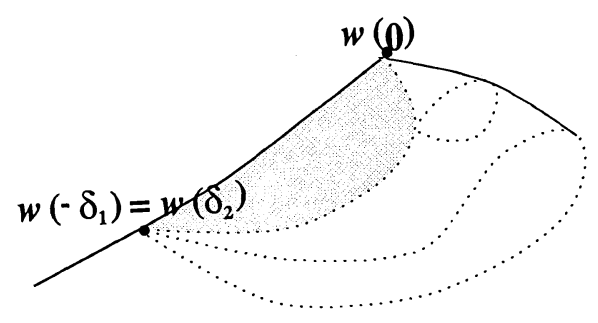

Figure 5.

Lemma 3.5.1. Under the hypotheses as in Proposition 3.5, there cannot be any pair of collar neighborhoods $U$ of $\left(-\delta_{1}, 0\right)$ and $V$ of $\left(0, \delta_{2}\right)$ for some $0<\delta_{1}, \delta_{2}<\min \left\{\epsilon_{1}, \epsilon_{2}\right\}$ such that

$$
w_{i}(U)=w_{j}(V)
$$

for any $i \neq j, 1 \leq i, j \leq 2$.

Proof. Because of (iii), $w_{i}$ are immersed on $\left(-\epsilon_{i}, \epsilon_{i}\right) \backslash\{0\}$ for $i=1$, 2. Suppose the contrary that there exist such neighborhoods $U, V$ and assume without loss of any generality that

$$
w_{1}(U)=w_{2}(V)
$$


Since self-intersection points can accumulate only at critical points, there exist some $0<\delta_{1}^{\prime}<\widetilde{\delta}_{1}<\delta_{1}$ and $0<\delta_{2}^{\prime}<\widetilde{\delta}_{2}<\delta_{2}$ and collar neighborhoods $A, B$ of $\left(-\widetilde{\delta}_{1},-\delta_{1}^{\prime}\right)$ and $\left(\delta_{2}^{\prime}, \widetilde{\delta}_{2}\right)$ in $D_{\epsilon_{1}}(0)$ and $D_{\epsilon_{2}}(0)$ respectively such that $\left.w_{1}\right|_{A}$ and $\left.w_{2}\right|_{B}$ are one-one and

$$
w_{1}(A)=w_{2}(B), \quad w_{1}\left(-\delta_{1}^{\prime}\right)=w_{2}\left(\delta_{2}^{\prime}\right), \quad w_{1}\left(-\widetilde{\delta}_{1},-\delta_{1}^{\prime}\right)=w_{2}\left(\delta_{2}^{\prime}, \widetilde{\delta}_{2}\right) .
$$

Then we consider the map $\left.\left(\left.w_{1}\right|_{A}\right)^{-1} \circ w_{2}\right|_{B}: B \rightarrow A$. Because $\left(\left.w_{1}\right|_{A}\right)^{-1} \circ$ $\left.w_{2}\right|_{B}: B \rightarrow A$ is holomorphic and so preserves the complex orientations of $B, A \subset \mathbb{C}$, the diffeomorphism

$$
\left.\left(\left.w_{1}\right|_{\left(-\widetilde{\delta}_{1},-\delta_{1}^{\prime}\right)}\right)^{-1} \circ w_{2}\right|_{\left(\delta_{2}^{\prime}, \widetilde{\delta}_{2}\right)}:\left(\delta_{2}^{\prime}, \widetilde{\delta}_{2}\right) \rightarrow\left(-\widetilde{\delta}_{1},-\delta_{1}^{\prime}\right)
$$

must preserve the induced (boundary) orientations which are nothing but the standard orientations on them. However this is impossible by Corollary 2.7 because we assume $w_{1}\left(-\delta_{1}^{\prime}\right)=w_{2}\left(\delta_{2}^{\prime}\right)$ from (ii) and hence this finishes the proof.

Lemma 3.5.2. Under the same hypotheses as in Proposition 3.5, there exist some $\delta_{1}, \delta_{2}>0$ such that

$$
w_{1}\left(\left(0, \delta_{1}\right)\right)=w_{2}\left(\left(0, \delta_{2}\right)\right)
$$

Proof. Suppose the contrary, say, that there exist a sequence of points $x_{j} \in$ $\left(0, \epsilon_{1}\right)$ with $x_{j} \rightarrow 0$ such that

$$
w_{1}\left(x_{j}\right) \notin w_{2}\left(\left(0, \epsilon_{2}\right)\right) .
$$

By the continuity of $w_{1}$ and by (v) for a sufficiently large $j_{0}$, we can choose a smooth curve $c_{0}:[0,1] \rightarrow D_{\epsilon_{1}}(0)$ such that

$$
\begin{aligned}
& c_{0}(0) \in\left(-\epsilon_{1}, 0\right), \quad c_{0}(1)=x_{j_{0}} \\
& c_{0}(t) \in \operatorname{Int} D_{\epsilon_{1}}(0) \backslash w_{1}^{-1}\left(w_{2}\left(\partial D_{\epsilon_{2}^{\prime}}(0)\right)\right) \quad \text { and } \\
& \operatorname{dist}\left(p, w_{1}\left(c_{0}(t)\right)\right) \leq \frac{d}{2} \quad \text { for } 0<t<1 .
\end{aligned}
$$

It follows from (3.2) and (v) that

$$
w_{1}\left(c_{0}\right) \cap w_{2}\left(\overline{D_{\epsilon_{2}}} \backslash D_{\epsilon_{2}}\right)=\emptyset .
$$


Define

$$
t_{0}=\sup \left\{t \in[0,1] \mid w_{1}\left(c_{0}(t)\right) \in \operatorname{Im} w_{2}\right\}
$$

From (iv), the main hypothesis on the collar neighborhoods $U_{1}, U_{2}$ and hypothesis (3.1), it follows that

$$
0<t_{0}<1
$$

By the continuity of $w_{2}$ and from (3.3) we derive that

$$
w\left(c_{0}\left(t_{0}\right)\right)=w_{2}(z) \quad \text { for some } z \in D_{\epsilon_{2}}(0) .
$$

From (3.2), $z \in \operatorname{Int} D_{\epsilon_{2}^{\prime}}(0)$. Since $d w_{1}\left(c_{0}\left(t_{0}\right)\right) \neq 0 \neq d w_{2}(z)$ from (iii), we can choose a small disc $B_{\delta}(z) \subset$ Int $D_{\epsilon_{2}}(0)$ by Lemma 2.5 such that $w_{1} \circ \phi=w_{2}$ on $B_{\delta}(z)$ for a holomorphic map $\phi: B_{\delta}(z) \rightarrow$ Int $B_{\epsilon_{1}}(0)$. Therefore we can find $t_{1}>t_{0}$ such that $w_{1}\left(c_{0}\left(t_{1}\right)\right) \subset \operatorname{Im} w_{2}$ which contradicts the definition of $t_{0}$. Hence, we have proved Lemma 3.5.2.

Proof of Proposition 3.5. Again by choosing sufficiently small $\widetilde{\delta}_{i}$ 's, we may assume that the map

$$
\left.\left(\left.w_{1}\right|_{\left(0, \widetilde{\delta}_{1}\right)}\right)^{-1} \circ w_{2}\right|_{\left(0, \widetilde{\delta}_{2}\right)}:\left(0, \widetilde{\delta}_{2}\right) \rightarrow\left(0, \widetilde{\delta}_{1}\right)
$$

becomes a diffeomorphism. Since $w_{1}(0)=w_{2}(0)$ from (ii), this diffeomorphism must preserve the orientations on $\left(0, \widetilde{\delta}_{i}\right)$ 's. Therefore Proposition 3.5 immediately follows from Corollary 2.7 (ii) and Lemma 2.5, which finishes the proof.

The next proposition shows that even when the boundary image of one disc penetrates into the interior image of the other, overlap of the image uniquely propagates across the arms of certain asterisk, at least near the center of the asterisk. We recommend readers to see Figure 6 before reading the statement of this proposition.

Proposition 3.7. Let $w:\left(D_{\epsilon}(0), \partial D_{\epsilon}(0)\right) \rightarrow(M, R)$ be a J-holomorphic map with $d w(z) \neq 0$ on $D_{\epsilon}(0) \backslash\{0\}$, and let $C=\bigcup_{j=1}^{k+2} \lambda_{j} \subset D_{\epsilon}(0)$ be $a(k+2)$ armed semi-asterisk in $D_{\epsilon}(0)$ centered at 0 such that $w(C) \subset R$ and $\left.w\right|_{C \backslash\{0\}}$ is an embedding (away from a discrete set of points). And let $u: \Delta \rightarrow M$ be a J-holomorphic map such that all the points in $(w(C) \backslash w(0)) \cap \operatorname{Im} u$, if any, are regular values of $u$. 
Suppose that there exists a collar neighborhood $U_{1}$ of $\lambda_{1}$ and an arc $\delta_{1} \subset$ $\Delta$ and a collar neighborhood $V_{1}$ of $\delta_{1}$ such that

$$
w\left(\lambda_{1}\right)=u\left(\delta_{1}\right) \text { and } w\left(U_{1}\right)=u\left(V_{1}\right) .
$$

Then there exists a set of arcs $\delta_{1}, \delta_{2}, \ldots, \delta_{k+2}$ in $\Delta$ that share one of end points from each, and collar neighborhoods $U_{\ell}, V_{\ell}$ of $\lambda_{\ell}^{\prime} \subset \lambda_{\ell}$ and $\delta_{\ell}$ respectively such that

$$
w\left(\lambda_{\ell}^{\prime}\right)=u\left(\delta_{\ell}\right) \text { and } w\left(U_{\ell}\right)=u\left(V_{\ell}\right)
$$

for all $1 \leq \ell \leq k+2$. Note that in particular the union $\bigcup_{j=1}^{k+2} \delta_{j}$ is connected, and that some of $\delta_{j}$ 's could be overlapped with one another.
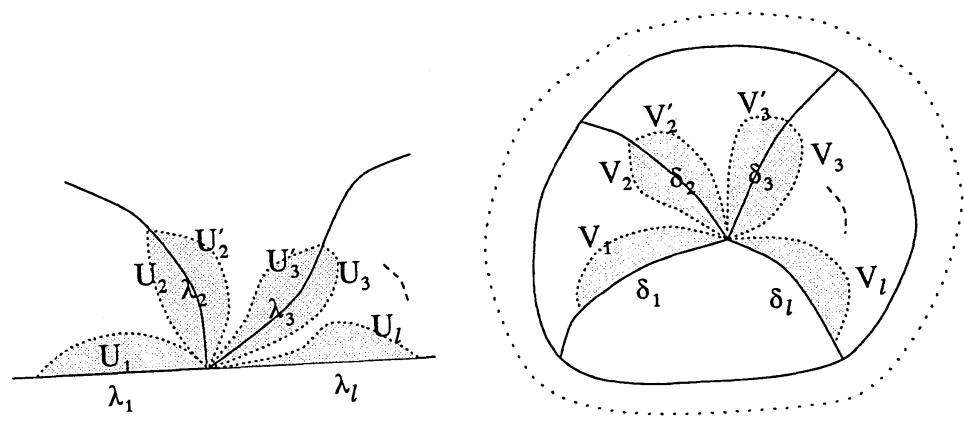

Figure 6.

Proof. When $k=0, C=\left(-\epsilon_{1}, 0\right] \cup\left[0, \epsilon_{2}\right)$ for some $0<\epsilon_{i}<\epsilon$. From (3.4) and Corollary 3.3, there exists $0<\epsilon_{2}^{\prime} \leq \epsilon_{2}$ and a collar neighborhood $N$ of $\left(-\epsilon_{1}, \epsilon_{2}^{\prime}\right)$ in $D_{\epsilon}(0)$ such that

$$
w(N) \subset \operatorname{Im} u .
$$

Consider the subset $u^{-1}(w(N)) \subset \Delta$. Certainly this contains the collar neighborhood $V_{1} \cap u^{-1}(w(N))$ of $\delta_{1}$ in $\Delta$.

Let $z_{1} \in \Delta$ be one of the end points of $\delta_{1}$ in $\Delta$ such that $w(0)=u\left(z_{1}\right)$. It will be enough to prove that the connected component of $u^{-1}(w(C \cap N))$ containing $\delta_{1}$ contains a non-trivial connected arc $\delta_{2}$ having $z_{1}$ as one end point. Then it will follow

$$
u\left(\delta_{2}\right)=w\left(\left(0, \epsilon_{2}^{\prime \prime}\right)\right)
$$


for some $0<\epsilon_{2}^{\prime \prime}<\epsilon_{2}^{\prime}$ and so (3.5) for $\ell=2$. However this immediately follows from the fact that $u: B_{\delta}\left(z_{1}\right) \backslash\left\{z_{1}\right\} \rightarrow M$ is immersed for some $\delta>0$, and so $u: B_{\delta}\left(z_{1}\right) \rightarrow \operatorname{Im} u$ is a branched covering and an open map. This finishes the proof of Proposition 3.7 for $k=0$.

Now suppose that we have proven the proposition for $1 \leq \ell \leq k+1$, and consider the case $\ell=k+2$. Consider the asterisk $C^{\prime}=C \backslash\left\{\lambda_{k+2}\right\}$ and apply the Riemann-mapping theorem to make $C^{\prime}$ into a $(k+1)$-armed semi-asterisk. Applying the induction hypothesis, there exists a sequence of $\left\{\delta_{\ell}^{\prime}\right\}_{1 \leq \ell \leq k+1}$ consisting of connected arcs sharing one of the end points from each such that there exist sub-arcs $0 \in \lambda_{\ell}^{\prime} \subset \lambda_{\ell}$

$$
w\left(\lambda_{\ell}^{\prime}\right)=u\left(\delta_{\ell}^{\prime}\right)
$$

and collar neighborhoods $U_{\ell}, V_{\ell}$ of $\lambda_{\ell}^{\prime}$ and $\delta_{\ell}$ respectively with

$$
w\left(U_{\ell}\right)=u\left(V_{\ell}\right) .
$$

Finally consider the 2-armed asterisk $C^{\prime \prime}=\lambda_{k+1} \cup \lambda_{k+2} \subset D_{\epsilon}(0)$. First note that the collar neighborhood $U_{k+1}$ obtained above lie in the side towards the arm $\lambda_{k}$ as drawn in Figure 7.

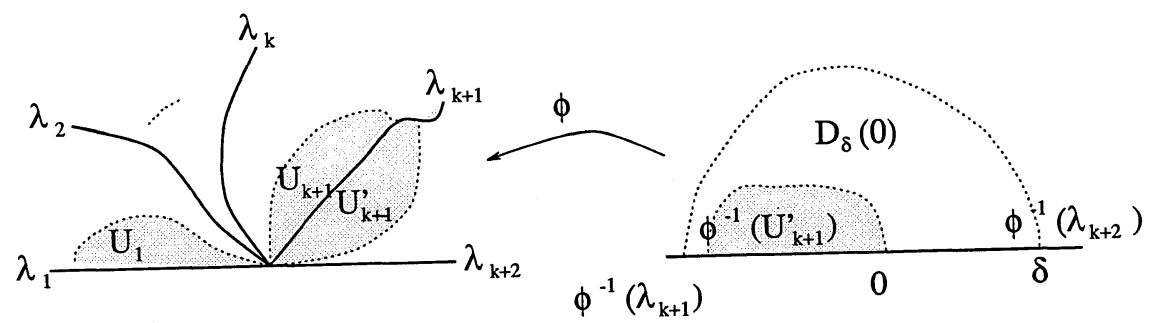

Figure 7.

We extend the collar neighborhoods $U_{k+1}$ and $V_{k+1}$ to bi-collar neighborhoods of $\lambda_{k+1}^{\prime}$ and $\delta_{k+1}$ respectively to obtain another collar neighborhoods $U_{k+1}^{\prime}$ and $V_{k+1}^{\prime}$ of $\lambda_{k+1}^{\prime}$ and $\delta_{k+1}$ respectively in the other sides, which still satisfy (3.5) but this time $U_{k+1}^{\prime}$ lie in between the arms $\lambda_{k+1}$ and $\lambda_{k+2}$. Now we apply the Riemann-mapping theorem to this region and denote the corresponding Riemann map by $\phi$ and then the composition 
$w \circ \phi:\left(D_{\delta}(0), \partial D_{\delta}(0)\right) \rightarrow(M, R)$ and $u$ satisfy the hypothesis of Proposition 3.5 , and so we can choose $\delta_{k+2}, \lambda_{k+2}^{\prime}$ and $U_{k+2}, V_{k+2}$ satisfying all the requirements by applying Proposition 3.5. This finishes the proof.

\section{Proof of Theorem I and II.}

We analyze structure of the set $\Delta \backslash w^{-1}(b A)$ in this section. When $\Delta \backslash w^{-1}(b A)$ is connected and simply conncected, Theorem I [O4] gives a complete answer and so we assume that $\Delta \backslash w^{-1}(b A)$ has more than one connected component or has at least one non-smply connected component. As a first step, we prove that $\Delta \backslash w^{-1}(b A)$ has only finitely many connected components. The following lemma shows that if there is any accumulation point of self-intersections, the limit point must be a critical point. The case in which the limit point is in the interior was studied in [M1], and so we will consider only the case where the limit point lies in the boundary $\partial D^{2}$ of $D^{2}$.

Lemma 4.1. Let $w:\left(D^{2}, \partial D^{2}\right) \rightarrow(M, R)$ be a J-holomorphic map, $z \in$ $\partial D^{2}$ and $\lambda$ be an open arc in $\partial D^{2}$ containing $z$, in its interior. We fix a sufficiently small collar neighborhood $U$ of $\lambda$. Assume that there is a sequence $\left\{x_{k}\right\} \subset w\left(\partial D^{2}\right) \cap w(\operatorname{Int} U)$ such that $\lim _{k \rightarrow \infty} x_{k}=w(z)$. Then we must have

$$
d w(z)=0 .
$$

Proof. Suppose the contrary that $d w(z) \neq 0$. By applying Lemma 2.4 and considering local pieces of $w$ near $z$, we may assume $(M, R)=\left(\mathbb{C}^{n}, \mathbb{R}^{n}\right)$ and

$$
u: D_{\epsilon}(0) \rightarrow \mathbb{C}^{n}, u(z)=(z, 0, \ldots, 0), J(z, 0, \ldots, 0)=i .
$$

Furthermore, there is a given sequence $\left\{z_{\mu}\right\} \subset \operatorname{Int} D_{\epsilon}(0)$ such that

$$
u\left(z_{\mu}\right) \in \mathbb{R}^{n}, \quad \lim _{\mu \rightarrow \infty} z_{\mu}=0, \quad z_{\mu} \neq 0 .
$$

But $u(z) \notin \mathbb{R}^{n}$ for $z \notin \partial D_{\epsilon}(0)$. This gives rise to a contradiction.

Unlike the case of closed curves (see [M4], [Si]), it is unlikely that the number of self-intersection points of discs is finite in general. (See some examples in $[\mathrm{C}]$ from which we believe one can construct a counter example).

Denote by $X \subset D^{2}$ the set of critical points. We recall the multiplicity function

$$
\kappa: A \rightarrow \mathbb{Z}_{+}
$$


and denote

$$
\begin{aligned}
A_{k} & =\{a \in A \mid \kappa(a)=k\} \text { and } \\
S & =D^{2} \backslash w^{-1}\left(A_{1}\right) .
\end{aligned}
$$

We call $S$ the set of self-intersection points of $w$ and denote by $S_{0} \subset S$ the subset of isolated self-intersection points of at least two different branches of the image.

Lemma 4.1 together with the corresponding results for the interior in [M1] implies that $S_{0} \backslash X$ is a discrete subset of $D^{2}$. We next quote a useful result from [O4].

Lemma 4.2 [Lemma 4.4, O4]. There exists a positive integer $M>0$ such that

$$
1 \leq \kappa(a)<M<\infty
$$

for all $a \in b A$ (and so for all $a \in A$ ).

Since the "vertices" of the "net" $\Delta \backslash w^{-1}(b A)$ must be critical points of $w$ in $\Delta$ and the number of different branches of $\Delta \cap w^{-1}(b A)$ at each vertex must be finite by Lemma 4.2 , we have the following immediate corollary of the above discussion

Corollary 4.3. $\Delta \backslash w^{-1}(b A)$ has only a finite number of connected components.

We now denote

$$
\Delta \backslash w^{-1}(b A)=\coprod_{j=1}^{k} E_{j}
$$

where $\left\{E_{j}\right\}_{j=1}^{k}$ is the set of connected components. Note that the isolated points in $\cup_{j=1}^{k} \bar{E}_{j} \backslash E_{j}$ are exactly

$$
S_{0} \cap w^{-1}(b A)=: S_{0}^{\prime} .
$$

Here we denote by $\bar{E}_{j}$ the closure of $E_{j}$ in $D^{2}$, not in $\Delta$. We add the points in $S_{0}^{\prime}$ to $E_{j}$ to define

$$
\widetilde{E}_{j}=E_{j} \cup\left(S_{0}^{\prime} \cap \operatorname{Int} \bar{E}_{j}\right) \subset \Delta .
$$

Then we have

$$
\left(\Delta \backslash w^{-1}(b A)\right) \cup S_{0}^{\prime}=\coprod_{j=1}^{k} \widetilde{E}_{j}
$$


where $\widetilde{E}_{j}$ are open subsets of $D^{2}$ which are domains in $\mathbb{C}$ without discrete holes in its interior.

Remark. The boundaries of $\widetilde{E}_{j}$ are not simple closed circles, but finite unions of simple closed circles and closed arcs attached in general. Moreover, an inner boundary can be a finit union of closed arcs only.
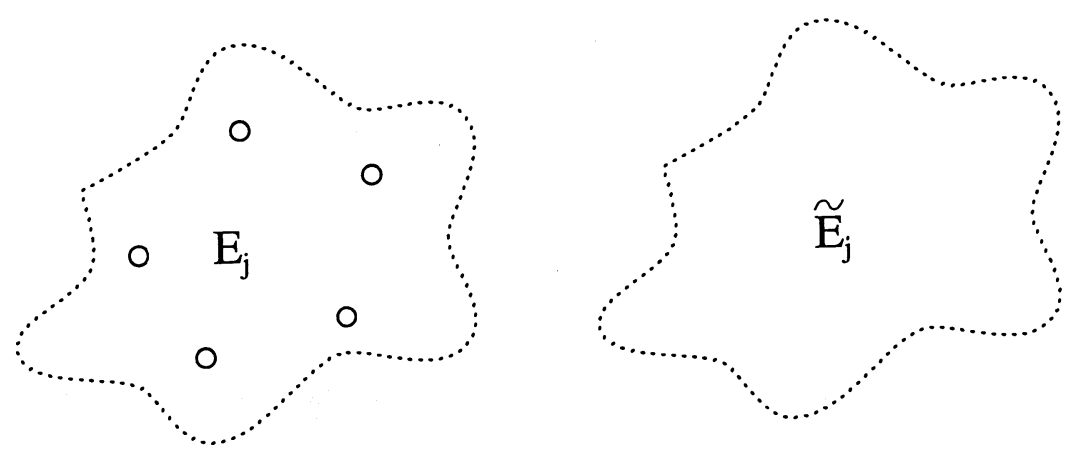

Figure 8.

The following example shows that in general $\widetilde{E}_{j}$ may not be simply connected even after adding a discrete set of points. (See [Example 2.4, La])

Example. Consider the complex plane $\mathbb{C}$ with coordinates $z=x+i y$. We identify $S^{2}=\mathbb{C} \cup\{\infty\}$. Let $B \subset S^{2}$ be the closed disc with the radius $1 / 2$ centered at $1+i$ and let $D$ be the complement to the interior of $B$ in $S^{2}$.

Then we consider a map $F: S^{2} \rightarrow S^{2}, F(z)=z^{4}$ and let $w$ be its restriction to $D$. Denote by $\Gamma$ Image $\left.w\right|_{\partial B}$. Note that $\Gamma$ is a smooth embedded circle in $S^{2}$ and hence a totally real submanifold in $S^{2}$. For the map $w:(D, \partial D) \rightarrow(M, R)$ where $M=S^{2}$ and $R=\Gamma$, it is easy to check that $D \backslash w^{-1}(\Gamma)$ contains a connected component which is non-simply connected (even after adding a discrete set of points).

With this example in mind, we prove the following theorem. We will postpone proof of the statement on the homology class to Appendix 3.

Theorem 4.4. In (4.3), we can further decompose, if necessary, each domain $\widetilde{E}_{j}$ into the finite union $\cup_{i} \widetilde{E}_{j}^{i}$ so that $\widetilde{E}_{j}^{i}$ are simply connected and so $\left.w\right|_{\widetilde{E}_{j}^{i}}$ can be reduced to a map $w_{j}^{i}:\left(D^{2}, \partial D^{2}\right) \rightarrow(M, R)$ with $\operatorname{Im} w_{j}^{i}=$ 
$\left.\operatorname{Im} w\right|_{\widetilde{E}_{j}}$ and

$$
\left[\left.w\right|_{\widetilde{E}_{j}}\right]=\sum_{i}\left[w_{j}^{i}\right] \quad \text { in } \quad H_{2}(M, R ; \mathbb{Z}) .
$$

Before giving the proof of this theorem, let us give the proof of Theorem II.

Proof of Theorem II (assuming Theorem 4.4). From Theorem 4.4, it follows that

$$
\text { Image }\left.w\right|_{\Delta}=\bigcup_{j=1}^{k} \bigcup_{i=1}^{n_{j}}\left(\text { Image }\left.w\right|_{\widetilde{E}_{j}^{i}}\right)
$$

where $\widetilde{E}_{j}^{i}$ 's can be identified with simply connected domains as in (4.16). For each given $\widetilde{E}_{j}^{i}$, we choose a Riemann-map $\phi_{j}^{i}: D^{2} \rightarrow \widetilde{E}_{j}^{i}$ and consider the composition $w_{j}^{i}:=w \circ \phi_{j}^{i}: D^{2} \rightarrow M$ which is continuous up to the boundary. Since the Riemann-map $\phi_{j}^{i}: D^{2} \rightarrow \widetilde{E}_{j}^{i}$ is proper i.e., maps $\partial D^{2}$ to $\partial E_{j}^{i}$ and $w\left(\partial D^{2}\right) \subset R$, we have $w_{j}^{i}\left(\partial D^{2}\right) \subset R$. Then by the boundary regularity theorem (see [O1] or [Y1]) for $J$-holomorphic discs with totally real boundary condition, $w_{j}: D^{2} \rightarrow M$ is smooth up to the boundary and defines a smooth $J$-holomorphic map

$$
w_{j}^{i}:\left(D^{2}, \partial D^{2}\right) \rightarrow(M, R)
$$

Furthermore, $D^{2} \backslash\left(w_{j}^{i}\right)^{-1}\left(w_{j}^{i}\left(\partial D^{2}\right)\right)$ is connected and simply conncected by the construction of $\widetilde{E}_{j}^{i}$ and $w_{j}^{i}$. Now we apply Theorem 1.1 to $w_{j}^{i}$ to conclude that there exists a Blaschke product $b_{j}: D^{2} \rightarrow D^{2}$ such that

$$
w_{j}^{i}=\widetilde{w}_{j}^{i} \circ b_{j}^{i}
$$

with $\widetilde{w}_{j}^{i}:\left(D^{2}, \partial D^{2}\right) \rightarrow(M, R)$ is a simple map. Combining the above discussion, we have finished the proof of Theorem II.

Now, the remaining section will be spent to prove Theorem 4.4 except the statement on the homology class. There are two main steps in the proof: the first is the step of decomposing the domain $\widetilde{E}_{j}$ into

$$
\widetilde{E}_{j}=\cup \widetilde{E}_{j}^{i}
$$

so that $\widetilde{E}_{j}^{i}$ are simply connected, and the other is the step of reducing the image $\left.w\right|_{\widetilde{E}_{j}^{i}}$ to that of a map from the disc $D^{2}$. 
Suppose that at least one of $\widetilde{E}_{j}$ 's is not simply connected. Then we can find $\widetilde{E}_{j_{0}}$, by taking an innermost one, such that its boundary $\partial \widetilde{E}_{j_{0}}$ is not connected. Then it will

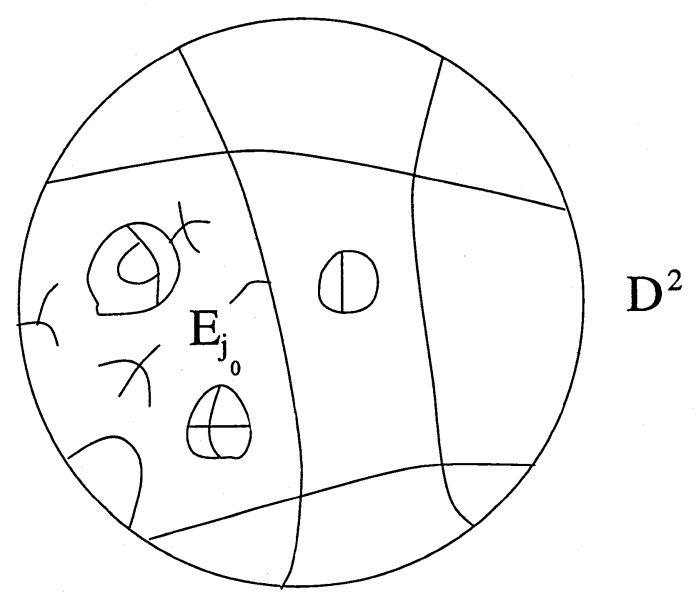

Figure 9.

i.e., consist of one outer boundary and a finite number of inner boundaries contained in $\Delta$ where each inner boundary contains, if any, only simply connected $\widetilde{E}_{k}$ 's in its interior. Note that some inner boundary may not have interior, and not be equal to $\cup_{k} \partial \widetilde{E}_{k}$ where $\widetilde{E}_{k}$ 's are the domains contained in the inside of the inner boundary. See Figure 9.

\subsection{Step I: Decomposition.}

For the convenience of notation, we assume $E_{1}=E_{j_{0}}$. Denote by $B_{1}$ the outer boundary and $\left\{B_{2}^{i}\right\}_{i=1}^{m}$ be the set of connected components of the inner boundary.

In this subsection, we will prove the following result.

Proposition 4.5. There exist mutually disjoint connected collar neighborhoods $U_{B_{1}}, V_{B_{2}^{i}}$ of $B_{1}$ and $B_{2}^{i}$ respectively such that

$$
\overline{\widetilde{E}_{1}}=\left(\bar{U}_{B_{1}} \cup\left(\cup_{i} \bar{V}_{B_{2}^{i}}\right)\right)
$$

and

$$
w\left(\bar{U}_{B_{1}}\right)=w\left(\bar{V}_{B_{2}^{i}}\right)=w\left(\overline{\widetilde{E}_{1}}\right)
$$


for all $i$. Furthermore we have

$$
\partial \bar{U}_{B_{1}}=B_{1} \coprod C_{1} \partial \bar{V}_{B_{2}^{i}}=B_{2}^{i} \coprod C_{2}^{i},
$$

where $C_{1}, C_{2}^{i} \subset \widetilde{E}_{1}$ are some connected union of piecewise smooth arcs contained in $\widetilde{E}_{1}$ (See Figure 15).

We take partitions

$$
\partial D^{2} \backslash X=\coprod_{i=1}^{s} \lambda_{i}, B_{1} \backslash X=\coprod_{j=1}^{t} \mu_{j}, B_{2}^{i} \backslash X=\coprod_{k} \delta_{k}^{i}
$$

where $\lambda_{i}, \mu_{j}$ and $\delta_{k}^{i}$ are connected open arcs without critical points of $w$ and the orderings are taken clockwise. By refining the partitions further if necessary, we may assume that any pair from $w\left(\lambda_{i}\right)$ 's, $w\left(\mu_{i}\right)$ 's and $w\left(\delta_{k}^{i}\right)$ 's is either identical or disjoint except possibly away from a discrete set of intersections. We have

$$
\text { Interior of } B_{2}^{i}=\coprod_{j=1}^{k_{i}}\left(\widetilde{\widetilde{E}_{i, j}} \backslash B_{2}^{i}\right) \quad\left(k_{i} \geq 0\right)
$$

where $\widetilde{E}_{i, j}$ 's are the domains inside $B_{2}^{i}$ among the set of $\widetilde{E}_{k}$ 's. We denote

$$
F^{i}=B_{2}^{i} \cup\left(\bigcup_{j=1}^{k_{i}} \partial \widetilde{E}_{i, j}\right) .
$$

By the choice of $E_{1}$, it follows that each $F^{i}$ must be connected. 


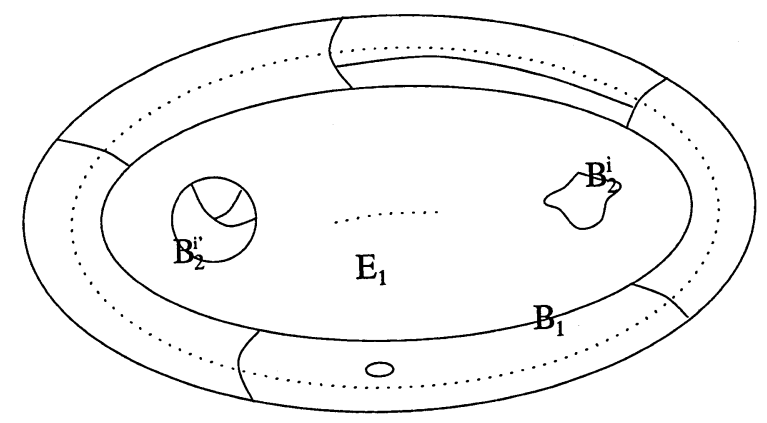<smiles>[10BH]C1=CCCCC1</smiles><smiles>Fc1ccccc1</smiles>

Figure 10.

Now consider a (collar-)neighborhood $U$ of $\partial D^{2}$ in $D^{2}$ and open neighborhoods $V^{i}$ of $\bigcup_{j=1}^{k_{i}} \widetilde{E}_{i, j}$ such that $U$ and $V^{i}$ are all disjoint and $\left(U \cap w^{-1}(b A)\right) \backslash S_{0}^{\prime}$ consists of disjoint open arcs one or both of whose two ends lie in $\partial D^{2}$. See Figure 11 below.

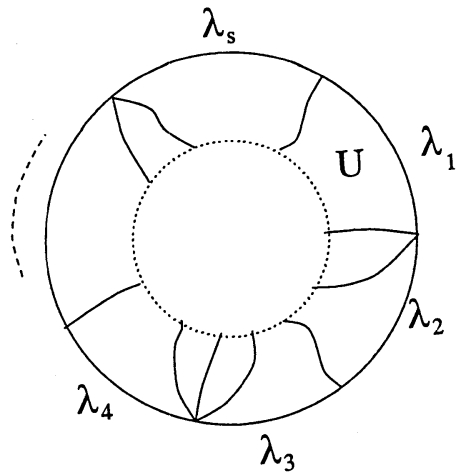

Figure 11.

Since $w\left(B_{2}^{i}\right) \subset b A=w\left(\partial D^{2}\right)$, there exist $\lambda \in\left\{\lambda_{j}\right\}_{j=1}^{s}, \delta^{i} \in\left\{\delta_{k}^{i}\right\}$ and collar neighborhoods $U_{1} \subset U$ and $V_{1}^{i} \subset V^{i}$ of $\lambda$ and $\delta^{i}$ respectively such that they satisfy

$$
w\left(U_{1}\right)=w\left(V_{1}^{i}\right), w(\lambda)=w\left(\delta^{i}\right) \subset w\left(B_{2}^{i}\right) \subset w\left(F^{i}\right) .
$$

We would like to note that the collar neighborhood $V_{1}^{i}$ may not be contained in $E_{1}$ but in $\widetilde{E}_{i, j_{i}}$ for some $j_{i} \in\left\{1,2, \cdots, k_{i}\right\}$. 
Claim I. $b A=w\left(\partial D^{2}\right)=w\left(F^{i}\right)$.

Proof. Obviously, $w\left(F^{i}\right) \subset b A$ and so it is enough to prove

$$
b A\left(=w\left(\partial D^{2}\right)\right) \subset w\left(F^{i}\right) .
$$

By cyclically re-ordering $\left\{\lambda_{j}\right\},\left\{\delta_{j}^{i}\right\}$ if necessary, we assume $\lambda=\lambda_{1}$ and $\delta^{i}=\delta_{1}^{i}$.

The claim immediately follows from the following lemma which proves that the overlap in (4.6) propagates along all the components $\left\{\lambda_{j}\right\}_{j=1}^{s}$ through.

Lemma 4.6. Let $i_{0}$ be fixed. For each $\lambda_{k}$, there is an arc $\delta_{k}^{i_{0}} \subset F^{i_{0}}$ and collar neighborhoods $U_{k}$ and $V_{k}^{i}$ of $\lambda_{k}$ and $\delta_{k}^{i_{0}}$ respectively such that

$$
w\left(U_{k}\right)=w\left(V_{k}^{i_{0}}\right)
$$

Proof. We will prove this by induction. For $k=1$, it follows from (4.6). Suppose we have proven it for any $1 \leq \ell \leq k$ and consider the case for $k+1$. We consider a small collar neighborhood $W$ of $\overline{\lambda_{k}} \cup \overline{\lambda_{k+1}}$ in $U$ and the neighborhood $V^{i_{0}}$ chosen above.

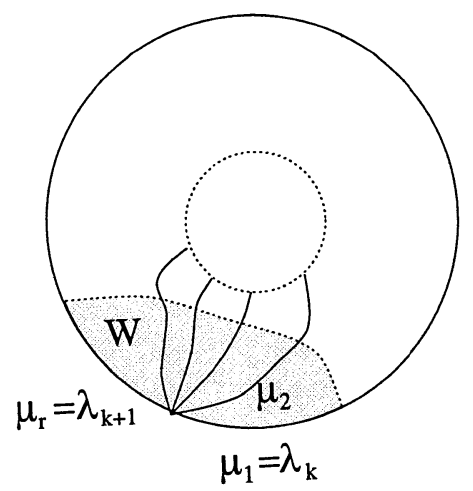

Figure 12.

In general, $W \cap w^{-1}(b A)$ becomes an $r$-armed (semi)-asterisk $C$ with $\lambda_{k}$ and $\lambda_{k+1}$ as the initial and the final arms respectively (see Figure 12). We 
order these arms $\left\{\mu_{j}\right\}_{j=1}^{r}$ counterclockwise with $\mu_{1}=\lambda_{k}, \mu_{r}=\lambda_{k+1}$. We now apply Proposition 3.7 to the maps

$$
\left.w\right|_{(W, \partial W)} \text { and } w: V^{i_{0}} \rightarrow M
$$

and find a sequence of arcs, $\nu_{1}, \cdots, \nu_{r} \subset V^{i_{0}}$, sub-arcs $\mu_{j}^{\prime} \subset \mu_{j}$ and collar neighborhoods $V_{j}^{\prime}$ and $U_{j}^{\prime}$ of $\nu_{j}$ and $\mu_{j}^{\prime}$ respectively such that

$$
w\left(\nu_{j}\right)=w\left(\mu_{j}^{\prime}\right) \text { and } w\left(U_{j}^{\prime}\right)=w\left(V_{j}^{\prime}\right)
$$

and $\nu_{j}$ 's share one of the end points from each and in particular

$$
\bigcup_{j=1}^{r} \nu_{j} \text { is connected. }
$$

Since $\nu_{1}=\delta_{1}^{i_{0}} \subset F^{i_{0}}$ and $F^{i_{0}}$ is a connected component of $w^{-1}(b A)$, it follows that

$$
\bigcup_{j=1}^{r} \nu_{j} \subset F^{i_{0}} .
$$

Note that due to the way how we partitioned in (4.4), we can extend $\mu_{r}^{\prime}$ and $\nu_{r}$ correspondingly $V_{r}^{\prime}$ and $U_{r}^{\prime}$ until $\mu_{r}^{\prime}$ becomes a full edge of $\mu_{r}=\lambda_{k+1}$ so that

$$
w\left(\nu_{r}\right)=w\left(\lambda_{k+1}\right), w\left(V_{r}^{\prime}\right)=w\left(U_{r}^{\prime}\right)
$$

in $F^{i_{0}}$, we have proven the existence of $\gamma_{k+1}^{i_{0}}, U_{k+1}$ and $V_{k+1}$ required as in Lemma 4.6 by setting $V_{r}^{\prime}=V_{k+1}$ and $U_{r}^{\prime}=U_{k+1}$.

Note that since $\widetilde{E}_{1} \cap w^{-1}(b A)$ and $\widetilde{E}_{i, j} \cap w^{-1}(b A)$ are at most discrete, we can apply the unique continuation for the interior image (Lemma 2.5) to $\left.w\right|_{\widetilde{E}_{1}}$ and $\left.w\right|_{\widetilde{E}^{i, j}}$ to derive the following lemma.

Lemma 4.7. If there are open sets $U \subset \widetilde{E}_{1}$ and $V \subset \widetilde{E}_{i, j}$ such that $w(U)=$ $w(V)$, then

$$
w\left(\widetilde{E}_{1}\right)=w\left(\widetilde{E}_{i, j}\right)
$$

In particular,

$$
w\left(\partial \widetilde{E}_{1}\right)=w\left(\partial \widetilde{E}_{i, j}\right) .
$$

Using Claim I and Lemma 4.7, we can choose disjoint collar neighborhoods of $B_{1}$ and $B_{2}^{i}$ in $\widetilde{E}_{1}$ with the same image (see Figure 14).

Claim II. For each $i$, we can choose disjoint collar neighborhoods $U_{B_{1}}$ and $V_{B_{2}^{i}}$ of $B_{1}$ and $B_{2}^{i}$ respectively such that

$$
\operatorname{Int} U_{B_{1}} \cup \operatorname{Int} V_{B_{2}^{i}} \subset \widetilde{E}_{1} \quad \text { and } \quad w\left(U_{B_{1}}\right)=w\left(V_{B_{2}^{i}}\right) .
$$


Proof. First note that $w\left(B_{1}\right) \subset b A=w\left(F^{i}\right)$ by Claim I for any $i$. Therefore for any $\mu_{j} \in\left\{\mu_{j}\right\}_{j=1}^{t}$, there exists an arc $\gamma_{j} \subset F^{i} \backslash X$ such that there are collar neighborhoods $U_{j} \subset \widetilde{\widetilde{E}_{1}}$ and $V_{j}^{i}$ of $\mu_{j}$ and $\gamma_{j}$ respectively with

$$
w\left(U_{j}\right)=w\left(V_{j}^{i}\right) \text { and } w\left(\mu_{j}\right)=w\left(\gamma_{j}\right) .
$$

Here Int $V_{j}^{i}$ may either be contained in $\widetilde{E}_{1}$ or in $\widetilde{E}_{i, \ell_{j}}$ for some $\ell_{j}$, as we mentioned above. But, we now prove that we can choose them so that

$$
\gamma_{j} \subset B_{2}^{i} \quad \text { and } \quad \operatorname{Int} V_{j}^{i} \subset \widetilde{E}_{1} .
$$

Assume that $V_{j}^{i} \subset \overline{\widetilde{E}_{i, \ell_{j}}}$.

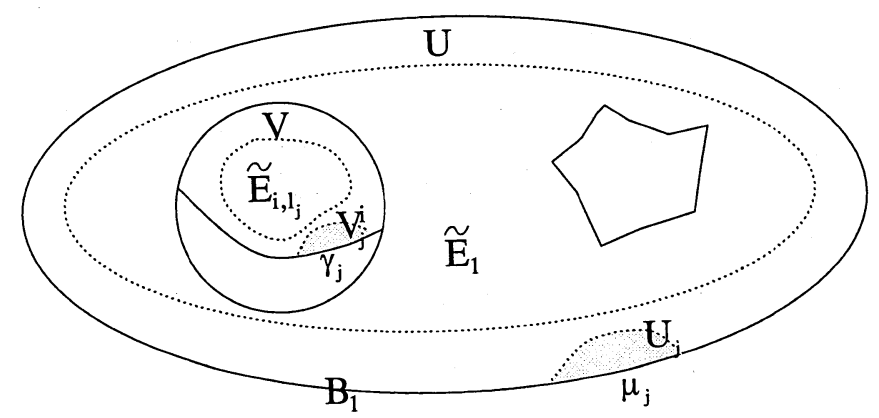

Figure 13.

By applying Proposition 3.5 to $\left.w\right|_{\widetilde{E}_{1}}$ and $\left.w\right|_{\widetilde{E}_{i, \ell_{j}}}$ repeatedly along $B_{1}$ and $\partial \widetilde{E}_{i, \ell_{j}}$, we get collar neighborhoods $U$ and $V$ of $B_{1}$ and $\partial \widetilde{E}_{i, \ell_{j}}$ respectively such that

$$
\operatorname{Int} U \subset \widetilde{E}_{1}, \quad \text { Int } V \subset \widetilde{E}_{i, \ell_{j}} \quad \text { and } \quad w(U)=w(V) .
$$

In particular,

$$
w\left(B_{1}\right)=w\left(\partial \widetilde{E}_{i, \ell_{j}}\right)
$$

And, by Lemma 4.7,

$$
w\left(\widetilde{E}_{1}\right)=w\left(\widetilde{E}_{i, \ell_{j}}\right) \quad \text { and } \quad w\left(\partial \widetilde{E}_{1}\right)=w\left(\partial \widetilde{E}_{i, \ell_{j}}\right) .
$$


Since $B_{2}^{i} \subset \partial \widetilde{E}_{1}$, from (4.9) and (4.10),

$$
w\left(B_{2}^{i}\right) \subset w\left(B_{1}\right) .
$$

Moreover, from (4.10), we can choose an $\operatorname{arc} \widetilde{\gamma}_{j} \subset B_{2}^{i}$ and a collar neighborhood $\widetilde{V}_{j}^{i} \subset{\widetilde{E_{1}}}_{1}$ of $\widetilde{\gamma}_{j}$ such that

$$
w\left(\widetilde{\gamma}_{j}\right)=w\left(\gamma_{j}\right)=w\left(\mu_{j}\right) \quad \text { and } \quad w\left(\widetilde{V}_{j}^{i}\right)=w\left(V_{j}^{i}\right)=w\left(U_{j}\right) .
$$

Hence comes the proof of (4.8).

Now, we apply Proposition 3.5 to $\left.w\right|_{\widetilde{E}_{1}}$ repeatedly along $B_{1}$ and $B_{2}^{i}$, then we get collar neighborhoods $U_{B_{1}}$ and $V_{B_{2}^{i}}$ of $B_{1}$ and $B_{2}^{i}$ respectively such that

$$
\operatorname{Int} U_{B_{1}} \cup \operatorname{Int} V_{B_{2}^{i}} \subset \widetilde{E}_{1} \quad \text { and } \quad w\left(U_{B_{1}}\right)=w\left(V_{B_{2}^{i}}\right) \text {. }
$$

By taking $U_{B_{1}}$ and $V_{B_{2}^{i}}$ smaller if necessary, we may assume that they are disjoint from one another. This finishes the proof of Claim II.

By applying Claim II for each $i$, we can choose disjoint collar neighborhoods $U_{B_{1}}$ and $V_{B_{2}^{i}}$ of $B_{1}$ and $B_{2}^{i}$ in $\widetilde{E}_{1}$ respectively such that

$$
\begin{aligned}
& w\left(U_{B_{1}}\right)=w\left(V_{B_{2}^{i}}\right) \\
& \operatorname{Int} U_{B_{1}} \cup \operatorname{Int} V_{B_{2}^{i}} \subset \widetilde{E}_{1} \\
& \left.w\right|_{\widetilde{E}_{1}} ^{-1}\left(\left.w\right|_{\widetilde{E}_{1}}\left(U_{B_{1}}\right)\right)=U_{B_{1}} \cup\left(\cup_{i} V_{B_{2}^{i}}\right) .
\end{aligned}
$$

Here the last condition is possible because the multiplicity of $\kappa_{\widetilde{E}_{1}}: w\left(\widetilde{E}_{1}\right) \rightarrow$ $\mathbb{Z}$ is constant (on an open dense subset of $\widetilde{E}_{1} \backslash X$ ) from the construction of $\widetilde{E}_{1}$.

Denote by $\bar{U}_{B_{1}}$ be the closure of $U_{B_{1}}$ in $\widetilde{E}_{1}$. If $\bar{U}_{B_{1}} \cup\left(\cup_{i} \bar{V}_{B_{2}^{i}}\right) \neq \widetilde{E}_{1}$, there exists $z_{0} \in \bar{U}_{B_{1}}$ with $d w\left(z_{0}\right) \neq 0$ such that $B_{\epsilon}\left(z_{0}\right) \cap\left(\cup_{i} \bar{V}_{B_{2}^{i}}\right)=\emptyset$ for some small $\epsilon>0$. (Note that $w\left(\bar{U}_{B_{1}}\right)=w\left(\bar{V}_{B_{2}^{i}}\right)$.) 


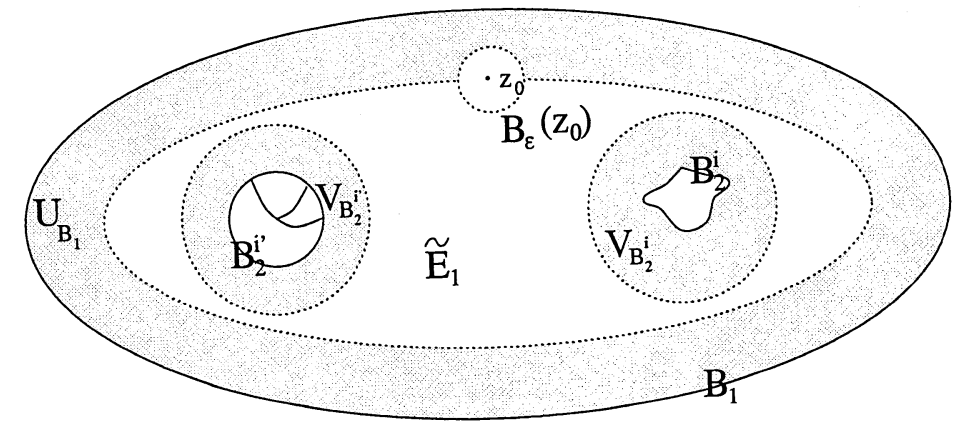

Figure 14 .

Then for any $z \in w^{-1}\left(w\left(z_{0}\right)\right)$ with $z \in \overline{\left(U_{B_{1}} \cup\left(\cup_{i} V_{B_{2}^{i}}\right)\right)} \backslash\left(U_{B_{1}} \cup\left(\cup_{i} V_{B_{2}^{i}}\right)\right)$ there exists some $\delta=\delta_{z}>0$ such that $B_{\delta_{z}}(z)$ has intersection with only one of the elements in $\left\{\bar{U}_{B_{1}}, \bar{V}_{B_{2}^{i}}\right\}$. Therefore, we can analytically continue $U_{B_{1}}$ and $V_{B_{2}^{i}}$ across $z_{0} \in \bar{U}_{B_{1}}$ and each $z \in w^{-1}\left(w\left(z_{0}\right)\right)$ in $\cup_{i} \bar{V}_{B_{2}^{i}}$ so that

(i) there exists some $\epsilon>0, B_{\epsilon}\left(z_{0}\right) \cap\left(\cup_{i} \bar{V}_{B_{2}^{i}}\right)=\emptyset$.

(ii) $U_{B_{1}}, V_{B_{2}^{i}}$ are still mutually disjoint

(iii) $w\left(U_{B_{1}}\right)=w\left(V_{B_{2}^{i}}\right)$ for all $i$

(iv) $w^{-1}\left(w\left(U_{B_{1}}\right)\right)=U_{B_{1}} \cup\left(\cup_{i} V_{B_{2}^{i}}\right)$.

By taking a maximal such extension of $U_{B_{1}}$ and $V_{B_{2}^{i}}$, we may conclude that there are mutually disjoint connected collar neighborhoods $U_{B_{1}}, V_{B_{2}^{i}}$ of $B_{1}$ and $B_{2}^{i}$ respectively such that

$$
\overline{\widetilde{E}_{1}}=\left(\bar{U}_{B_{1}} \cup\left(\cup_{i} \bar{V}_{B_{2}^{i}}\right)\right)
$$

and

$$
w\left(\bar{U}_{B_{1}}\right)=w\left(\bar{V}_{B_{2}^{i}}\right)=w\left(\widetilde{\widetilde{E}}_{1}\right)
$$

for all $i$. Moreover, we have

$$
\partial \bar{U}_{B_{1}}=B_{1} \coprod C_{1} \partial \bar{V}_{B_{2}^{i}}=B_{2}^{i} \coprod C_{2}^{i},
$$


where $C_{1}, C_{2}^{i} \subset \widetilde{E}_{1}$ are some connected union of closed piecewise smooth arcs contained in $\widetilde{E}_{1}$ (See the following example). This finishes the proof of Proposition 4.5.

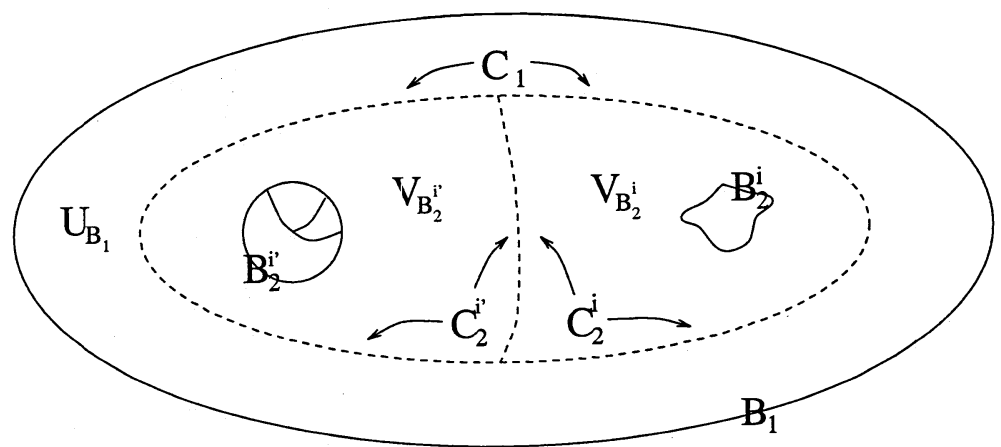

Figure 15.

\subsection{Step II: Reduction.}

For the notational convenience, we denote

$$
C_{2}^{0}=C_{1}, B_{2}^{0}=B_{1}, V_{B_{2}^{0}}=U_{B_{1}}
$$

and

$$
C_{2}^{i} \backslash\left(X \cup S_{0}\right)=\coprod_{j} \lambda_{j}^{i} .
$$

Note that since $C_{2}^{i}$ is a compact subset of $\Delta=\operatorname{Int} D^{2}, C_{2}^{i} \cap\left(X \cup S_{0}\right)$ is finite by the result in [M4], the above union is a finite union.

Lemma 4.8. Let $i$ be fixed. For each $\lambda_{j}^{i}$, there is $\lambda_{k_{j}}^{i}$ which is different from $\lambda_{j}^{i}$ such that

$$
w\left(\lambda_{j}^{i}\right)=w\left(\lambda_{k_{j}}^{i}\right)
$$

and $\left.\left(\left.w\right|_{\lambda_{k_{j}}^{i}}\right)^{-1} \circ w\right|_{\lambda_{j}^{i}}: \lambda_{j}^{i} \rightarrow \lambda_{k_{j}}^{i}$ reverses the induced orientations. 
Proof. Note that $\lambda_{j}^{i}$ is shared by $V_{B_{2}^{i}}$ and $V_{B_{2}^{i^{\prime}}}$ for some $i^{\prime} \neq i$. Let $V^{i} \subset$ $V_{B_{2}^{i^{\prime}}} \subset \widetilde{E}_{1} \backslash V_{B_{2}^{i}}$ be a collar neighborhood of $\lambda_{j}^{i}$. Since $w\left(V_{B_{2}^{i}}\right)=w\left(V_{B_{2}^{i^{\prime}}}\right)$ and so $w\left(C_{2}^{i}\right)=w\left(C_{2}^{i^{\prime}}\right)$, there exist some $k_{j}$ and a collar neighborhood $U^{k_{j}} \subset V_{B_{2}^{i}}$ of $\lambda_{k_{j}}^{i}$ such that $w\left(V^{i}\right)=w\left(U^{k_{j}}\right)$. By the local injectivity of $w$ applied to a regular point $z_{1} \in \lambda_{j}^{i}$, it follows that from Corollary 2.7 that the map $\left.\left(\left.w\right|_{\lambda_{k_{j}}^{i}}\right)^{-1} \circ w\right|_{\lambda_{j}^{i}}: \lambda_{j}^{i} \rightarrow \lambda_{k_{j}}^{i}$ must reverse the induced orientation.

Note that $\lambda_{k_{j}}^{i}$ may not be unique in general. We now fix $\ell$ and denote by $\delta_{\ell}^{i}$ an $\operatorname{arc} \lambda_{k_{\ell}}^{i}$ corresponding to $\lambda_{\ell}^{i}$ as in Lemma 4.6. Due to (4.11) and to the choice of $\delta_{\ell}^{i}$, for a given collar neighborhood $U_{\ell}^{i} \subset \bar{V}_{B_{2}^{i}}$ of $\lambda_{\ell}^{i}$, there must ' exist a collar neighborhood $V_{\ell}^{i} \subset \bar{V}_{B_{2}^{i^{\prime}}}$ for some $i^{\prime} \neq i$ such that

$$
w\left(U_{\ell}^{i}\right)=w\left(V_{\ell}^{i}\right) .
$$

By continuing $\left.w\right|_{V_{\ell}^{i}}$ across $\delta_{\ell}^{i}$ into $B_{2}^{i}$, we can find a (bi-collar) neighborhood $W_{\ell}^{i}$ of $\delta_{\ell}^{i}$ so that

$$
W_{\ell}^{i} \backslash V_{\ell}^{i} \subset V_{B_{2}^{i}}
$$

and the images of $\left.w\right|_{U_{\ell}^{i}}$ and $\left.w\right|_{W_{\ell}^{i} \backslash V_{\ell}^{i}}$ match smoothly along $w\left(\lambda_{\ell}^{i}\right)=w\left(\delta_{\ell}^{i}\right)$.

We call a sequence $\lambda_{1}^{i}, \cdots \lambda_{\ell}^{i}$ a chain, provided that

(1) each consecutive pair $\overline{\lambda_{k}^{i}}, \overline{\lambda_{k+1}^{i}}$ have a common boundary point $x_{\lambda k}, k=$ $1, \cdots, \ell-1$

(2) $\left\{\delta_{1}^{i}, \cdots, \delta_{\ell}^{i}\right\}$ are as in Lemma 4.6 and and each consecutive pair $\overline{\delta_{k}^{i}}, \overline{\delta_{k+1}^{i}}$ share a common boundary point $x_{\delta k}, k=1, \cdots, \ell-1$

(3) the set $\left\{U_{1}^{i}, \cdots, U_{\ell}^{i}, V_{1}^{i}, \cdots, V_{\ell}^{i}\right\}$ can be chosen to be pairwise disjoint.

We denote by $x_{\lambda 0}$ (respectively $x_{\delta 0}$ ) the other end point of $\lambda_{1}^{i}$ (respectively $\delta_{1}^{i}$ ), by $x_{\lambda \ell}$ (respectively $x_{\delta \ell}$ ) the other end point of $\lambda_{\ell}^{i}$ (respectively $\delta_{\ell}^{i}$ ). Since there are only finite number of $\lambda_{j}^{i}$ 's, there exists a maximal chain.

Lemma 4.9. If $\left\{\lambda_{1}^{i}, \cdots, \lambda_{\ell}^{i}\right\}$ is a maximal chain, then

$$
\left(\cup \overline{\lambda_{j}^{i}}\right) \cup\left(\cup \overline{\delta_{j}^{i}}\right)=C^{i}
$$

and

$$
x_{\lambda 0}=x_{\delta 0}, \quad x_{\lambda \ell}=x_{\delta \ell}
$$


Proof. First note that if (4.14) holds, then it follows from the connectedness of $C^{i}$ that (4.13) must also hold. Suppose the contrary that at least one of the identities in (4.14) does not hold, say

$$
x_{\lambda \ell} \neq x_{\delta \ell} .
$$

It follows from (4.15) that there exists an arc $\lambda_{\ell+1}^{i} \subset C^{i} \backslash$ Let $\lambda_{\ell+1}^{i}$ be the arc attached to $x_{\lambda \ell}$ and $\delta_{\ell+1}^{i}$ be the corresponding arc chosen as in Lemma 4.6. We will show that $\delta_{\ell+1}^{i}$ can be attached to $x_{\delta \ell}$. If only two edges of $\left\{C_{2}^{i}\right\}$ meet at $x_{\delta \ell}$ we can choose collar neighborhoods $U_{\ell+1}^{i}, V_{\ell+1}^{i}$ of $\lambda_{\ell+1}^{i}, \delta_{\ell+1}^{i}$ such that

$$
w\left(U_{\ell+1}^{i}\right)=w\left(V_{\ell+1}^{i}\right), \quad \operatorname{Int} U_{\ell+1}^{i} \subset V_{B_{2}^{i}} \quad \text { and } \quad \operatorname{Int} V_{\ell+1}^{i} \subset V_{B_{2}^{i^{\prime}}}
$$

for some $i^{\prime}$ by a similar argument as in the proof of Proposition 3.5 (See Lemma 3.5.2). When more than two edges meet at $x_{\delta \ell}$, we can still apply Proposition 3.5 to the domain containing $V_{\ell+1}^{i}$ and prove that there is an $\operatorname{arc} \delta_{\ell+1}^{i^{\prime}}$ attached to $\overline{\delta_{\ell}^{i}}$ such that

$$
w\left(\overline{\lambda_{\ell}^{i}} \cup \overline{\lambda_{\ell+1}^{i}}\right)=w\left(\overline{\delta_{\ell}^{i}} \cup \overline{\delta_{\ell+1}^{i^{\prime}}}\right)
$$

and collar neighborhoods $U_{\ell+1}^{i}, V_{\ell+1}^{i^{\prime}}$ of $\lambda_{\ell+1}^{i}, \delta_{\ell+1}^{i^{\prime}}$ such that

$$
w\left(U_{\ell+1}^{i}\right)=w\left(V_{\ell+1}^{i^{\prime}}\right), \quad \operatorname{Int} U_{\ell+1}^{i} \subset V_{B_{2}^{i}} \quad \text { and } \quad \operatorname{Int} V_{\ell+1}^{i^{\prime}} \subset V_{B_{2}^{i^{\prime}}} .
$$

(See Figure 16.) Since $w\left(C_{2}^{i}\right)=w\left(C_{2}^{i^{\prime}}\right)$, there is an $\operatorname{arc} \delta_{\ell+1}^{i} \subset C_{2}^{i}$ attached to $\overline{\delta_{\ell}^{i}}$ such that

$$
w\left(\delta_{\ell+1}^{i}\right)=w\left(\delta_{\ell+1}^{i^{\prime}}\right)
$$

and collar neighborhood $V_{\ell+1}^{i}$ of $\delta_{\ell+1}^{i}$ such that

$$
w\left(V_{\ell+1}^{i^{\prime}}\right)=w\left(V_{\ell+1}^{i}\right) .
$$

It is easy to check that $\operatorname{Int} V_{\ell+1}^{i} \subset \widetilde{E}_{1} \backslash \bar{V}_{B_{2}^{i}}$. It follows that $\left\{\lambda_{1}^{i}, \cdots, \lambda_{\ell}^{i}, \lambda_{\ell+1}^{i}\right\}$ and $\left\{\delta_{1}^{i}, \cdots, \delta_{\ell}^{i}, \delta_{\ell+1}^{i}\right\}$ satisfy (1) and (2). We now show (3), which will contradict to maximality of the chain $\left\{\lambda_{1}, \cdots, \lambda_{\ell}^{i}\right\}$. If (3) fails, i.e., $U_{\ell+1}^{i}$ and $V_{\ell+1}^{i}$ cannot be chosen to be disjoint, we must have

$$
\lambda_{\ell+1}^{i}=\delta_{\ell+1}^{i} .
$$

Because of the definition of $\delta_{j}^{i}$ in Lemma 4.6, this implies that the image of $w$ must "fold" along this arc and so there must exist a critical point on 
this arc which contradicts that the arcs are free of critical points (See the beginning of Step II: Reduction). This-finishes the proof.

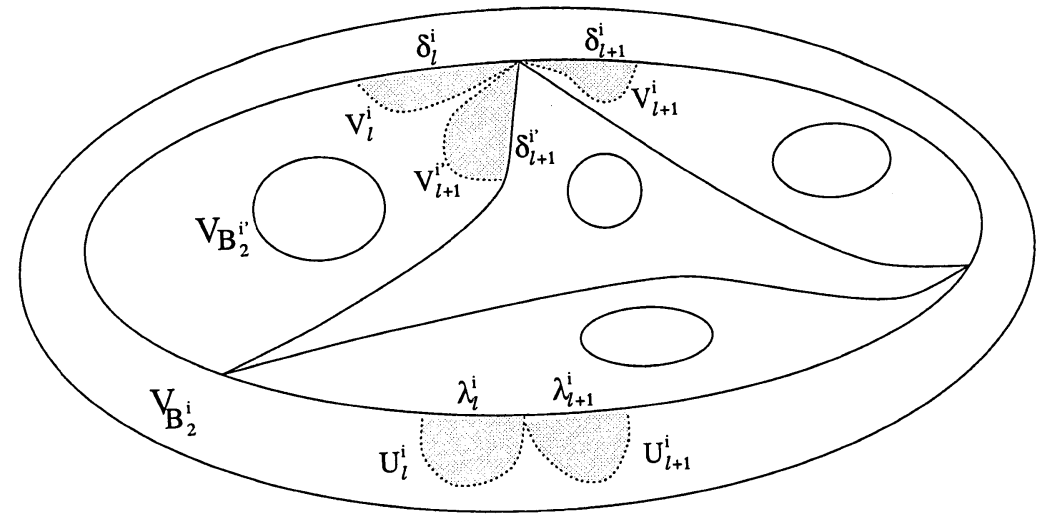

Figure 16.

We now define an equivalence relation $\sim$ on $\bar{V}_{B_{2}^{i}}$ : for $x \in \cup_{j=1}^{t} \lambda_{j}^{i}$ and $y \in \cup_{j=1}^{t} \delta_{j}^{i}$ by

$$
x \sim y \quad \text { iff } \quad x \in \lambda_{j}^{i}, y \in \delta_{j}^{i}, w(x)=w(y) .
$$

We may assume without loss of generality that $\partial V_{B_{2}^{i}}$ is a piecewise smooth simple closed circle. It follows from Lemma 4.9 that $D_{B_{2}^{i}}:=\bar{V}_{B_{2}^{i}} / \sim$ has a topological boundary $B_{2}^{i}$ and its interior is topologically a disc. Recall that for some neighborhoods $W_{j}^{i}$ of $\delta_{j}^{i}$ such that $W_{j}^{i} \backslash V_{j}^{i} \subset V_{B_{2}^{i}}, w\left(U_{j}^{i}\right)$ and $w\left(W_{j}^{i} \backslash \operatorname{Int} V_{j}^{i}\right)$ matches smoothly along $w\left(\lambda_{j}^{i}\right)$ and that $\left.\left(\left.w\right|_{\delta_{j}^{i}}\right)^{-1} \circ w\right|_{\lambda_{j}^{i}}: \lambda_{j}^{i} \rightarrow$ $\delta_{j}^{i}$ reverses the orientation. Therefore $\operatorname{Int} D_{B_{2}^{i}}$ has a complex structure away from at most finite possible singularities, which is induced from $\operatorname{Int} \bar{V}_{B_{2}^{i}}$. We extend the complex structure across the finite points and find a Riemann map

$$
\phi_{i}: \Delta \rightarrow \operatorname{Int} D_{B_{2}^{i}}
$$

which extends continuously up to the boundary.

We define a continuous map $\widetilde{w}^{i}: \operatorname{Int} \bar{V}_{B_{2}^{i}} / \sim \rightarrow M$ by

$$
\widetilde{w}^{i}([x])=w(x)
$$


which is $J$-holomorphic with respect to the induced complex structure on Int $\bar{V}_{B_{2}^{i}} / \sim$ away from the finite points, and which is continuous up to the boundary whose boundary image is contained in $R$. Finally we define the map $w^{i}: \Delta \rightarrow M$ by the composition

$$
w^{i}=\widetilde{w}^{i} \circ \phi^{i}
$$

This map is continuous up the boundary whose boundary image is contained in the totally real submanifold $R$, and $J$-holomorphic away from at most finite points. By the boundary regularity theorem and the removable singularity theorem [O1, Y1], $w^{i}$ becomes $J$-holomorphic everywhere on $\Delta$. and is smooth up to boundary. This finishes the proof of the following

Proposition 4.10. $\left.\left.w\right|_{\bar{U}_{B^{1}}} w\right|_{\bar{V}_{B_{2}^{i}}}$ can be reduced to maps from $\left(D^{2}, \partial D^{2}\right)$ to $(M, R)$ and in particular their images are the same as the images of $J$. holomorphic discs with boundary in $R$.

So far we have shown that by further decomposing the innermost nonsimply connected component $\overline{\widetilde{E}_{1}}$ into

$$
\bar{U}_{B_{1}} \cup\left(\bigcup_{i} \bar{V}_{B_{2}^{i}}\right),
$$

$\left.w\right|_{\bar{U}_{B_{1}}}$ and $\left.w\right|_{\bar{V}_{B_{2}^{i}}}$ 's can be reduced to maps from the unit disc according to the above equivalence relation (4.13). We note that

$$
\partial D_{B_{1}}=B_{1}=\text { the outer boundary of } \widetilde{E}_{1} .
$$

We glue $D^{2} \backslash \widetilde{E}_{1}$ and $D_{B_{1}}$ along $B_{1}$ and form the union $\left(D^{2} \backslash \widetilde{E}_{1}\right) \cup D_{B_{1}}$. This union has a naturally induced complex structure and is again topologically a disc. Hence it is biholomorphic to the standard disc. Since the above arguments applied $\widetilde{E}_{1}$ depends only on the fact that $\partial \widetilde{E}_{1}$ consists of one outer boundary and a finite number of inner boundaries such that each inner boundary only contains simply connected $\widetilde{E}_{k}$ 's in its inside, we can repeat the whole process to an innermost non-simply connected component, if any, of the union (4.14). This finally finishes the proof of Theorem 4.4 by setting $\widetilde{E}_{j}^{i}$ to be the discs we have obtained in (4.16).

In the above, we have used the connected region $D^{2} \backslash \widetilde{E}_{1}$ to glue with $D_{B_{1}}$ to produce a topological disc. 
Now let us choose, if any, the connected regions in $D^{2}$ which are inside of $B_{2}^{i}$ and have their boundaries in $B_{2}^{i}$ (see Figure 10). Consider $D^{i}$ that is the union of these regions and $\bar{V}_{B_{2}^{i}}$. This is a topologically disc with boundary $\partial \bar{V}_{B_{2}^{i}} \cap \widetilde{E}_{1}$. Hence, if we quotient $D^{i}$ by the equivalence relation (4.16), $D^{i} / \sim$ becomes a sphere $S^{2}$, which has a naturally induced complex structure. Moreover $w$ naturally induces a $J$-holomorphic map from the union, $u: S^{2} \rightarrow M$. Since $w\left(\bar{U}_{B_{1}}\right)$ and $w\left(\bar{V}_{B_{2}^{i}}\right)$ 's coincide as a set, the unique continuation theorem implies that the image of $w$ and $u$ coincide, i.e.,

$$
u\left(S^{2}\right)=w\left(D^{2}\right) .
$$

Hence, we get

Corollary 4.11. If one of $\widetilde{E}_{j}$ 's is not simply connected, then we can reduce $w$ to a map $u: S^{2} \rightarrow M$ such that Image $u=$ Image $w$. In particular, if $(M, J)$ (e.g., $\left.\mathbb{C}^{n}\right)$ does not admit a J-holomorphic sphere, all $\widetilde{E}_{j}$ becomes simply connected. In particular, when $(M, J)=\mathbb{C}^{n}$, all $\widetilde{E}_{j}$ are simply connected.

\section{Proof of Theorem III.}

By Theorem 4.4, we now know that each connected component $\widetilde{E}_{j}$ in $(\Delta \backslash$ $\left.w^{-1}(b A)\right) \cup S_{0}^{\prime}$ can be decomposed to $\cup_{i} \widetilde{E}_{j}^{i}$. We will abuse our notation to denote by $\widetilde{E}_{j}^{i}$ the discs we obtain in (4.16). In this section, we further study the finer structure of the "net" $w^{-1}(b A)$ and the image of $w:\left(D^{2}, \partial D^{2}\right) \rightarrow$ $(M, R)$.

Let $\phi_{j}^{i}: \Delta \rightarrow \widetilde{E}_{j}^{i}$ be a Riemann map and denote

$$
w_{j}^{i}=\left.w\right|_{\widetilde{E}_{j}^{i}} \circ \phi_{j}^{i}: \Delta \rightarrow M
$$

Since $\left.w\right|_{\widetilde{E}_{j}^{i}}$ is continuous up to boundary and so the Riemann map $\phi_{j}^{i}: \Delta \rightarrow$ $\widetilde{E}_{j}^{i}, w_{j}^{i}$ is continuous up to boundary with $w_{j}^{i}\left(\partial D^{2}\right) \subset R$. By the boundary regularity theorem, $w_{j}^{i}$ is smooth up to boundary and by the definition of $\widetilde{E}_{j}^{i}, w_{j}^{i}$ allows a factorization like (1.3). We denote the corresponding simple map by $\widetilde{w}_{j}^{i}$ and the Blaschke product by $b_{j}^{i}$, i.e.,

$$
w_{j}^{i}=\widetilde{w}_{j}^{i} \circ b_{j}^{i} .
$$

We first quote the following theorem proved in [O4], which describes structure of the images of simple maps. 
Proposition 5.1 [Theorem II, O4]. Let $\widetilde{w}:\left(D^{2}, \partial D^{2}\right) \rightarrow(M, R)$ be simple $J$-holomorphic map. Then the following alternative holds:

(i) there is a point $z \in \partial D^{2}$ such that

$$
\widetilde{w}^{-1}(\widetilde{w}(z)) \cap \partial D^{2}=\{z\} \quad \text { and } \quad D \widetilde{w}(z) \neq 0 .
$$

(ii) the multiplicity of $\kappa: b A \backslash X \rightarrow \mathbb{Z}_{+}$is two except at a discrete set of points where $X$ is the set of critical values. Furthermore, the image $A=$ Image $\widetilde{w}$ becomes a smooth immersed branched Riemann surface.

In particular, If $(M, J)$ does not carry any closed J-holomorphic Riemann surface (e.g. like $\mathbb{C}^{n}$ ), then only the alternative (i) must hold.

Definition 5.2. We call a simple map satisfying (i) type $I$ and one satisfying (ii) type II.

This is the main theorem we prove in this section.

Theorem 5.3. Let $\cup_{j=1}^{k} \widetilde{E}_{j}^{i}$ be the decomposition in Theorem 4.4, and $w_{j}^{i}, \widetilde{w}_{j}^{i}$ and $b_{j}^{i}$ defined as above, Then we have the alternative:

(i) all $\widetilde{w}_{j}^{i}$ 's are of type $I$

(ii) all $\widetilde{w}_{j}^{i}$ 's are of type II: In this case, the images of all $\widetilde{w}_{j}$ 's coincide as a set. Furthermore, in this case, the multiplicity of $\kappa: A \rightarrow \mathbb{Z}_{+}$is constant (on the open dense set of $A \backslash X$ ).

Proof. From Proposition 5.1, each $\widetilde{w}_{j}^{i}$ is either type I or type II. Suppose that there is at least one $\widetilde{w}_{j}^{i}$ of type II. Then since $\widetilde{w}_{j}^{i}$ is simple, we can decompose

$$
\partial \Delta_{j}^{i} \backslash X=\left(\cup_{k} \lambda_{k}\right) \cup\left(\cup_{k} \delta_{k}\right)
$$

so that $\left.\left(\left.\widetilde{w}_{j}\right|_{\delta_{k}}\right)^{-1} \circ \widetilde{w}_{j}\right|_{\lambda_{k}}: \lambda_{k} \rightarrow \delta_{k}$ is orientation reversing, where $\Delta_{j}^{i}=$ $\left(\phi_{j}^{i}\right)^{-1}\left(\widetilde{E}_{j}^{i}\right)$ and $X$ is the set of critical points.

The domains $\widetilde{E}_{j}^{i^{\prime}}$ which also come from of the decomposition of $\widetilde{E}_{j}$, satisfy

$$
w\left(\partial \widetilde{E}_{j}^{i}\right)=w\left(\partial \widetilde{E}_{j}^{i^{\prime}}\right) .
$$

It implies that $\Delta_{j}^{i^{\prime}}$ 's can be decomposed as (5.3) and hence $w_{j}^{i^{\prime}}$ 's are of type II. 
Assume that $\widetilde{E}_{k}^{i}$ is adjacent to $E_{j}$. Let $\partial \Delta_{k}^{i}=\cup_{\ell} t_{\ell}$ such that $\left.\widetilde{w}_{k}^{i}\right|_{t_{\ell}}$ is smooth. From the assumption that $\widetilde{E}_{k}^{i}$ is adjacent to $E_{j}$, in particular $E_{j}^{i_{0}}$ with the decomposition (5.3), there is some $t_{m}^{i}$ such that $\widetilde{w}_{k}^{i}\left(t_{m}\right)=$ $\widetilde{w}_{j}^{i_{0}}\left(\lambda_{m^{\prime}}\right)=\widetilde{w}_{j}^{i_{0}}\left(\delta_{m^{\prime}}\right)$. Since $\left.\left(\left.\widetilde{w}_{j}^{i_{0}}\right|_{\delta_{m^{\prime}}}\right)^{-1} \circ \widetilde{w}_{j}^{i_{0}}\right|_{\lambda_{m^{\prime}}}$ is orientation reversing, either $\left.\left(\left.\widetilde{w}_{k}^{i}\right|_{t_{m}}\right)^{-1} \circ \widetilde{w}_{j}^{i_{0}}\right|_{\lambda_{m^{\prime}}}$ or $\left(\left.\widetilde{w}_{k}^{i}\right|_{t_{m}}\right)^{-1} \circ \widetilde{w}_{j}^{i_{0}} \mid \delta_{m^{\prime}}$ must be orientation preserving. Without loss of any generality, we may assume $\left.\left(\left.\widetilde{w}_{k}^{i}\right|_{t_{m}}\right)^{-1} \circ \widetilde{w}_{j}^{i_{0}}\right|_{\lambda_{m^{\prime}}}$ is orientation preserving, i.e., there are collar neighborhoods $U_{m^{\prime}}$ of $\lambda_{m^{\prime}}$ in $\bar{\Delta}_{j}^{i_{0}}$ and $V_{m}$ of $t_{m}$ in $\bar{\Delta}_{k}^{i}$ such that

$$
\widetilde{w}_{j}^{i_{0}}\left(U_{m^{\prime}}\right)=\widetilde{w}_{k}^{i}\left(V_{m}\right)
$$

Applying Proposition 3.5 inductively along $\partial \Delta_{j}^{i_{0}}$ and $\partial \Delta_{k}^{i}$, we have

$$
\widetilde{w}_{j}^{i_{0}}\left(\partial \Delta_{j}^{i_{0}}\right)=\widetilde{w}_{k}^{i}\left(\partial \Delta_{k}^{i}\right) .
$$

So, we find an $\operatorname{arc} s_{m}$ in $\partial \Delta_{k}^{i}$ such that

$$
\left.\left(\left.\widetilde{w}_{k}^{i}\right|_{s_{m}}\right)^{-1} \circ \widetilde{w}_{j}^{i_{0}}\right|_{\delta_{j}}
$$

is orientation preserving. This implies that

$$
\widetilde{w}_{k}^{i}\left(t_{m}\right)=\widetilde{w}_{k}^{i}\left(s_{m}\right)
$$

Clearly, $t_{m}$ is not equal to $s_{m}$. Since $\widetilde{w}_{j}^{i_{0}}\left(\partial \Delta_{j}^{i_{0}}\right)=\widetilde{w}_{k}^{i}\left(\partial \Delta_{k}^{i}\right)$, for each arc component $t_{\ell}$ of $\partial \Delta_{k}^{i} \backslash X$, there is an arc component $s_{\ell} \neq t_{\ell}$ such that $\widetilde{w}_{k}^{i}\left(t_{\ell}\right)=\widetilde{w}_{k}^{i}\left(s_{\ell}\right)$. So we can conclude that $\widetilde{w}_{k}^{i}$ is also of type II.

By repeating this for each pair of adjacent domains, we have proven that all $\widetilde{w}_{j}^{i}$ must be of type II if there is one $\widetilde{w}_{j}^{i}$ of type II. The other statement in (ii) can be proven by similar arguments and hence the proof of Theorem 5.3 .

\section{The case of integrable $J$ and real-analytic $R$.}

In this section we restrict to the classical case in which the almost complex structure $J$ is integrable (at least near $R$ ) and the totally real submanifold $R$ is real-analytic, unless otherwise stated.

For the integrable $J$ and real-analytic $R$, we can refine Theorem 5.3 (i) as follows: 
Theorem 6.1. Suppose that $J$ is integrable near $R$ and $R$ is real-analytic. Then in the case of Theorem 5.3 (i), the union of the images of any two consecutive $\widetilde{w}_{j}^{i}, \widetilde{w}_{k}^{\ell}$ such that $j \neq k$ can be reduced to the image of a Jholomorphic sphere.

We first state several immediate corollaries of Theorem 5.3 and 6.1.

Corollary 6.2. We assume the same for $R$ and $J$ as in Theorem 6.1. For any $J$-holomorphic disc $w:\left(D^{2}, \partial D^{2}\right) \rightarrow(M, R)$, there always exist a simple disc $\widetilde{w}:\left(D^{2}, \partial D^{2}\right) \rightarrow(M, R)$ such that the image $\widetilde{w}$ is the same as that of $w$ as a set (without counting multiplicity).

Proof. If $w$ is already simple or the number of components in the decomposition (4.3) is 1 , then there is nothing to prove and so we assume that $w$ is not simple and the number of components is greater than 1 . In this case, $w$ must belong to one of the two cases in Theorem 5.3.

In the case of (ii), the image of $w$ coincide with the image of any $\widetilde{w}_{j}^{k}$. By composing this with the Riemann map from $\Delta$ to $\widetilde{E_{j}^{k}}$, we are done.

In the case of (i), we apply Theorem 6.1. Then for any two consecutive $\widetilde{w}_{i}^{j}, \widetilde{w}_{k}^{\ell}, i \neq k$, let $\widetilde{E}$ be a domain containing $\widetilde{E}_{i}^{j}, \widetilde{E}_{k}^{\ell}$ and some open arcs in $\partial \frac{\widetilde{E}_{i}^{j}}{\partial} \partial \overline{\widetilde{E}_{k}^{\ell}}$ so that $\widetilde{E}$ is simply connected. Since $w_{i}^{j}\left(\partial \widetilde{E}_{i}^{j}\right)=w_{k}^{\ell}\left(\partial \widetilde{E}_{k}^{\ell}\right)$, we may consider $\left.w\right|_{\widetilde{E}}$ as a map $w^{\prime}: S^{2} \rightarrow M$ which is $J$-holomorphic map. So, there is a map $\widetilde{w}^{\prime}: S^{2} \rightarrow M$ which is injective away from a set of points which is discrete in $S^{2} \backslash$ \{critical points\} and has the same image with $w^{\prime}$. Let $\lambda$ be a connected arc in $S^{2}$ such that $\widetilde{w}^{\prime}(\lambda) \subset R$. Now $\phi$ be a Riemann map $\Delta \rightarrow S^{2} \backslash \lambda$. Then $\widetilde{w}^{\prime} \circ \phi$ will be such a required map.

Note that for the map $w:\left(D^{2}, \partial D^{2}\right) \rightarrow\left(\mathbb{C}^{n}, R\right)$ with respect to an almost complex structure $J$ tamed with the standard symplectic structure, the alternative (ii) was ruled out in Proposition 5.1. Furthermore from Theorem 6.1, it also follows that there cannot be more than one connected component in the above decomposition, provided $J$ is integrable and $R$ is real-analytic. This immediately implies the following result which seems to be new in the classical context of several complex variables.

Corollary 6.3. Consider $\mathbb{C}^{n}$ with its standard complex structure $J_{0}$ and let $R$ be a real-analytic totally real submanifold in $\mathbb{C}^{n}$. Then for any $J_{0}$ holomorphic disc $w:\left(D^{2}, \partial D^{2}\right) \rightarrow\left(\mathbb{C}^{n}, R\right), \Delta \backslash w^{-1}(b A)$ is connected and simply connected (after adding a discrete set of points) and so allows the factorization (1.3). 
Remark 6.4. It follows from the example in the appendix that the result Theorem 6.1 fails to hold (and so Corollary 6.3, 6.5 and 6.7 below) for the smooth cases. The main obstruction comes from the fact that one cannot perform the reflection argument for non-integrable $J$ or not real-analytic $R$. This reflection principle is needed to prove Proposition 6.6 below. The example in the appendix also shows that Proposition 6.6 does not hold for the smooth cases.

Another interesting corollary of Theorem 6.1 is the structure of the "net" in the case of (i).

Corollary 6.5. Assume that $w:\left(D^{2}, \partial D^{2}\right) \rightarrow(M, R)$ satisfies $(i)$ in Theorem 5.3. Then the "net" $w^{-1}(\underset{\widetilde{E} A)}{ }$ allows a black-and-white coloring on $\Delta$ such that the images of $w_{j}$ from $\widetilde{E}_{j}$ 's with the same color are all the same as a set. In particular, all the interior nodes have even number of edges coming out.

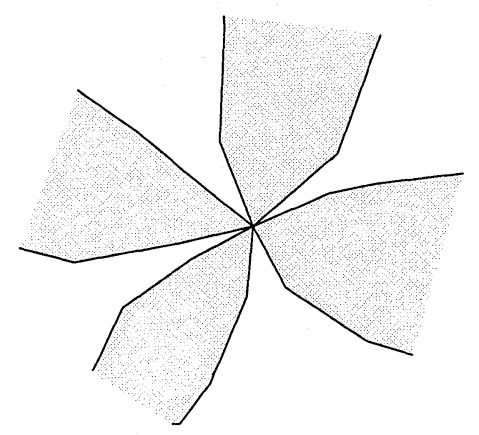

Figure 17.

The remaining section will be spent to prove Theorem 6.1.

Let $\bar{D}_{\epsilon}(0)$ be the semi-disk centered at 0 with boundary $(-\epsilon, \epsilon)$ in the lower half plane of $\mathbb{C}$. We will need to prove an analogue to Lemma 3.5.2 for the maps

$$
\begin{aligned}
& w_{1}:\left(D_{\epsilon}(0), \partial D_{\epsilon}(0)\right) \rightarrow(M, R) \\
& w_{2}:\left(\bar{D}_{\epsilon}(0), \partial \bar{D}_{\epsilon}(0)\right) \rightarrow(M, R)
\end{aligned}
$$

satisfying all the hypothesis on Proposition 3.5. 
Proposition 6.6. Let

$$
\begin{aligned}
& w_{1}:\left(D_{\epsilon_{1}}(0), \partial D_{\epsilon_{1}}(0)\right) \rightarrow(M, R) \\
& w_{2}:\left(\bar{D}_{\epsilon_{2}}(0), \partial \bar{D}_{\epsilon_{2}}(0)\right) \rightarrow(M, R)
\end{aligned}
$$

be J-holomorphic maps satisfying $(i)-(v)$ in Proposition 3.5. Suppose that there exist collar neighborhoods $U_{i}$ of $\left(-\epsilon_{i}, 0\right)$ in $D_{\epsilon_{i}}(0)$ with $\partial U_{i}=$ $\left(-\epsilon_{i}, 0\right)$ for $i=1,2$ and $\left.w_{i}\right|_{\partial U_{i}}$ is an embedding, and that the diffeomorphism $\left.\left(\left.w_{1}\right|_{\left(-\epsilon_{1}, 0\right)}\right)^{-1} \circ w_{2}\right|_{\left(-\epsilon_{2}, 0\right)}:\left(-\epsilon_{2}, 0\right) \rightarrow\left(-\epsilon_{1}, 0\right)$ preserves the orientations induced from the complex orientations on $D_{\epsilon_{1}}(0)$ and $\bar{D}_{\epsilon_{2}}(0)$ respectively. Then we can find collar neighborhoods $V_{i}$ of $\left(-\epsilon_{i}, \delta_{i}\right)$ such that $\delta_{i}>0$ and

$$
w_{1}\left(\partial V_{1}\right)=w_{2}\left(\partial V_{2}\right) \text {. }
$$

In particular, by Corollary 2.7 (ii), the union $w_{1}\left(V_{1}\right) \cup w_{2}\left(V_{2}\right)$ forms a smooth surface possibly with a branched point at 0 .

Proof. Since we assume that $J$ is integrable, $R$ is real-analytic and $w_{2}\left(\partial \bar{D}_{\epsilon_{2}}\right) \subset R$, we can apply the classical reflection principle to $w_{2}$ to get a map $\widetilde{w}_{2}:\left(D_{\epsilon_{2}}(0), \partial D_{\epsilon_{2}}(0)\right) \rightarrow(M, R)$. We can check that $w_{1}$ and $\widetilde{w}_{2}$ satisfy the assumptions in Proposition 3.5. So, from Proposition 3.5, there are $\delta_{i} \in\left(0, \epsilon_{i}\right)$ and collar neighborhoods $W_{i}$ of $\left(-\epsilon_{i}, \delta_{i}\right)$ for $i=1,2$ such that

$$
w_{1}\left(W_{1}\right)=\widetilde{w}_{2}\left(W_{2}\right) \text {. }
$$

Since $w_{2}\left(-\epsilon_{2}, \delta_{2}\right)=\widetilde{w}_{2}\left(-\epsilon_{2}, \delta_{2}\right)$, This finishes the proof.

From this proposition together with the argument used in the proof of $(4.8)$ or $(4,10)$, we get the following.

Corollary 6.7. Let $w_{j}^{i}: \widetilde{E}_{j}^{i} \rightarrow M$ and $w_{j^{\prime}}^{k}: \widetilde{E}_{j^{\prime}}^{k} \rightarrow M$ be two restriction of $w$ such that $\partial \widetilde{E}_{j}^{i}$ and $\partial \widetilde{E}_{j^{\prime}}^{k}$ share at least one edge. Then

(i)

$$
w_{j}^{i}\left(\partial \widetilde{E}_{j}^{i}\right)=w_{j^{\prime}}^{k}\left(\partial \widetilde{E}_{j^{\prime}}^{k}\right) .
$$

In particular, the union $B:=w_{j}^{i}\left(\partial \widetilde{E}_{j}^{i}\right)=w_{j^{\prime}}^{k}\left(\partial \widetilde{E}_{j^{\prime}}^{k}\right)$ becomes a compact closed branched Riemann surface.

(ii) The multiplicity of $B$ becomes one for the case of type $I$ and two for the case of type II.

Now, the proof of Theorem 6.1 can be achieved by applying Corollary 6.7 inductively with respect to an ordering of $\widetilde{E}_{j}^{i}$ by a connected chain of $\widetilde{E}_{j}^{i}$ 's. This finishes the proof of Theorem 6.1. 


\section{Appendix 1 (by Jean-Pierre Rosay).}

We show that the hypothesis of real analyticity of $R$ is needed in Proposition 6.6 (and so in Theorem 6.1, and Corollaries 6.3, 6.5, and 6.7.) The results do not generalize to the case when $R$ is only smooth. It is not completely clear whether the real analyticity of $R$ is needed for Corollary 6.2.

By $\Delta$ we denote the open unit disk, $b \Delta$ is the unit circle and $\bar{\Delta}=\Delta \cup b \Delta$. A (smooth) holomorphic disk in $\mathbb{C}^{2}$ is a smooth map from $\bar{\Delta}$ into $\mathbb{C}^{2}$, whose restriction to $\Delta$ is holomorphic.

Proposition A.1. There exists a non-constant (smooth) holomorphic dick in $\mathbb{C}^{2}$, and $M_{2}$ a 2-dimensional totally real manifold in $\mathbb{C}^{2}$ such that $f(b \Delta) \subset$ $M_{2}$ and $f^{-1}(f(b \Delta))$ disconnects $\Delta$.

Remark A.2. This cannot happen, by Corollary 6.3, if $M_{2}$ is real analytic.

Construction of $f$ and $M_{2}$.

1) Let $D^{+}$and $\Omega^{+}$be smooth by bounded, simply connected, regions in $\{\Im z>0\}$ (in $\mathbb{C}$ ) such that

$$
\left\{\begin{array}{l}
b D^{+} \cap \mathbb{R}=[-1,+1] \\
b \Omega^{+} \cap \mathbb{R}=[-1 / 2,+1 / 2]
\end{array}\right.
$$

Let $h^{+}$be a conformal map from $D^{+}$to $\Omega^{+}$, normalized so that $h^{+}[-1 / 2,1 / 2]=[-1 / 2,+1 / 2],\left(h^{+}\right.$extending smoothly to $\left.b D^{+}\right)$.

The main fact is that $\Im h^{+}=0$ on $[-1 / 2,+1 / 2]$ and $\Im h^{+}>0$ on $([-1,+1]-[-1 / 2,+1 / 2])$. 


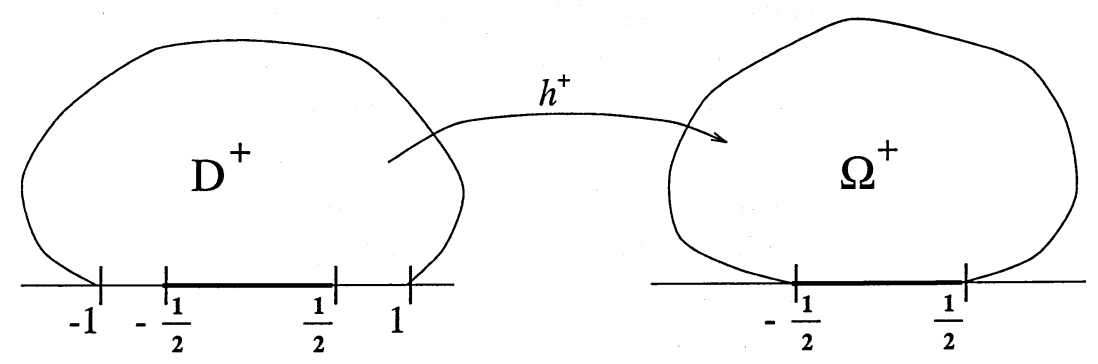

Figure 18.

2) Define $D^{-}, \Omega^{-}$by symmetry with respect to the real axis and $f^{-}$by Schwartz reflection:

$$
\begin{aligned}
D^{-} & =\left\{z \in \mathbb{C}, \bar{z} \in D^{+}\right\} \\
\Omega^{-} & =\left\{z \in \mathbb{C}, \bar{z} \in \Omega^{-}\right\} \\
h^{-}: D^{-} & \rightarrow \Omega^{-}, \quad h^{-}(z)=\overline{h^{+}(\bar{z}) .}
\end{aligned}
$$

3) Let $D=D^{+} \cup D^{-} \cup(-1 / 2,+1 / 2)$. By Schwartz reflection, there exists $h$ holomorphic on $D$ such that $h \mid D^{ \pm}=h^{ \pm}$. For later use, set also $h( \pm 1 / 2)=h^{+}( \pm 1 / 2)=h^{-}( \pm 1 / 2)$.

4) Construction of $M_{2}$.

Claim. There exists a smooth totally real manifold $M_{2}$ in $\mathbb{C}^{2}$ such that $\left(z, h^{+}\right)\left(b D^{+}\right)$and $\left(z, h^{-}\right)\left(b D^{-}\right) \subset M_{2}$. (Where $\left(z, h^{ \pm}\right)$denotes the maps $z \mapsto\left(z, h^{ \pm}(z)\right)$.

The only difficulty is at the points $x= \pm 1 / 2$, where $\left(x, h^{+}(x)\right)$ and $\left(x, h^{-}(x)\right)$ "bifurcate". Let $M_{2}^{0}=\left\{\left(x, h^{+}(x)\right)+i(0, t), \quad-1<x<\right.$ $+1, \quad t \in \mathbb{R}\}$. This is a smooth totally real manifold. Taking $t=0$, we see that it contains $\left(z, h^{+}\right)([-1,+1])$. Taking $t=-2 \Im h(x)$, we see that it contains $\left(z, h^{-}(z)\right)([-1,+1])$, since $h^{+}(x)-h^{-}(x)$ is purely imaginary, for $-1 \leq x \leq+1$. 
Extending $M_{2}^{0}$ to a totally real manifold containing $\left(z, h^{ \pm}\right)\left(b D^{ \pm}\right)$is now "soft". One can, for example, set, for $\epsilon$ small enough: $M_{2}=$ $M_{2}^{0} \cup M_{2}^{+} \cup M_{2}^{-}$.

$$
M_{2}^{ \pm}=\left\{\left(z, h^{ \pm}(z)\right)+i(0, t), \quad z \in b D^{ \pm}-(-1,+1), \quad-\epsilon<t<\epsilon\right\} .
$$

Note. The above construction shows that the real analyticity of $R$ is needed in Proposition 6.6.

5) End of construction.

Let $G$ be a conformal map from $\Delta$ into $D$. For simplicity impose $G(0)=0, G^{\prime}(0)>0$. This yields $G([0,1))=[0,1 / 2)$, by symmetry. Let $F$ be the holomorphic map from $\Delta$ into $\mathbb{C}^{2}$ defined by

$$
F(z)=\left(G\left(z^{2}\right), h\left(G\left(z^{2}\right)\right)\right) .
$$

Notice that $F$ extends continuously to $b \Delta$ by

$$
F(z)=\left(G\left(z^{2}\right), h^{ \pm}\left(G\left(z^{2}\right)\right)\right),
$$

\pm depending whether $\Im G\left(z_{1}^{2}\right) \geq 0$ or $\leq 0$ for $z_{1} \in \Delta, \quad z_{1}$ close to $z$ (if $G\left(z^{2}\right)= \pm 1 / 2, F(z)=( \pm 1 / 2, h( \pm 1 / 2))$. Since $z \in b \Delta$ implies that $G\left(z^{2}\right) \in b D, F(b \Delta) \subset M_{2}$. But also

$$
F([-1,+1])=\{(x, h(x)), 0 \leq x \leq 1 / 2\} \subset M_{2} .
$$

Let $\Delta_{1}=\Delta-[0,1]$. It follows from the above that $F\left(b \Delta_{1}\right)=$ $F(b \Delta \cup[0,1]) \subset M_{2}$. but since $F$ is even $F^{-1} F([0,1]) \supset[-1,+1]$. So $F^{-1}(F([0,1]))$ disconnects $\Delta_{1}$. Let $\psi$ be a conformal map from $\Delta$ onto $\Delta_{1}$. Set $f=F \circ \psi$. The pair $\left(f, M_{2}\right)$ gives the desired example.

Note. Although the smoothness of $f$ could be checked by following the construction, it also follows immediately from the continuity of $f$ and the fact that $f(b \Delta) \subset M_{2}\left(M_{2}\right.$ totally real, and smooth).

\section{Appendix 2.}

Rosay's example in Appendix 1 however still has constant multiplicity 2 and allows the factorization. In this appendix, we provide an example that has points of two different multiplicities and so does not allow the factorization. We will follow the same notations as in Appendix 1. 
We consider the map

$$
\chi: \Delta^{+} \rightarrow \Delta, \quad \chi(z)=z^{3}
$$

where $\Delta^{+}=\{z \in \Delta \mid \Im z>0\}$, the upper semi-disc. Note that

$$
\chi\left(b \Delta^{+}\right)=b \Delta \cup[-1,1] .
$$

Let $M_{2}$ be the totally real submanifold and $G$ be the map from $\Delta$ into $D$ in Appendix 1. Define the map $\widetilde{F}(z): \Delta^{+} \rightarrow \mathbb{C}^{2}$ by

$$
\widetilde{F}(z)=(G(\chi(z)), h(G(\chi(z)))) .
$$

Since $\chi\left(b \Delta^{+}\right)=b \Delta \cup[-1,1]$, we have correct boundary condition $\widetilde{F}\left(b \Delta^{+}\right) \subset$ $M_{2}$. Then it follows that $\widetilde{F}$ has points of two different multiplicities (in fact, 1 and 2). Composing $\widetilde{F}$ with a conformal map $\phi: \Delta \rightarrow \Delta^{+}$, we have obtained the seeked example $g=\widetilde{F} \circ \phi: \Delta \rightarrow \mathbb{C}^{2}$ with $g(b \Delta) \subset M_{2}$.

\section{Appendix 3.}

Since the decomposition in Theorem 4.4 uses cutting and pasting, it is not apriori clear that the homology class is preserved under decomposition. In this appendix, we prove that this is indeed the case.

Theorem A.3. The homology class is preserved under the decomposition in Theorem 4.4. (Theorem II).

Let $\widetilde{E}$ be the (innermost) non-simply connected component used in 4.1 (Decomposition). Let $B_{1}$ be the outer boundary and $\cup_{1}^{\ell} B_{2}^{i}$ be the inner boundaries of $\widetilde{E}$. Then $\cup_{1}^{\ell} B_{2}^{i}$ 's bound simply connected domains $G_{i}$ such that $\partial G_{i}=B_{2}^{i}$. As in 4.1 (decomposition), we decompose

$$
\widetilde{E}=\cup_{0}^{\ell} \widetilde{E}^{i}
$$

and denote by $w^{i}:\left(D^{2}, \partial D^{2}\right) \rightarrow(M, R)$ the reduction of $\left.w\right|_{\widetilde{E}^{i}}$ defined as in 4.2 (Reduction).

By the way how we obtain the clecomposition in Theorem 4.4, Theorem A.3 will be an immediate consequence of

Proposition A.4. Let $\widetilde{E}$ and $w^{i}$ be as above. Then we have

$$
\left[\left.w\right|_{E^{\prime}}\right]=\sum_{0}^{\ell}\left[w^{i}\right]
$$

in $H_{2}(M, R ; \mathbb{Z})$. 
Proof. As in 4.1 (decomposition) and 4.2 (reduction), we decompose

$$
\partial \widetilde{E}^{i}=B_{2}^{i} \coprod C^{i}
$$

where $C^{i} \subset \widetilde{C}^{i}$ and $B_{2}^{0}=B_{1}$ (See figure 15.)

Recall that in 4.2 (reduction), we have decomposed each $C^{i}$ into the union of two connected piecewise smooth arcs

$$
C^{i}=C_{\lambda}^{i} \cup C_{\delta}^{i}
$$

such that

$$
w\left(C_{\lambda}^{i}\right)=w\left(C_{\delta}^{i}\right)
$$

Furthermore we also have

$$
w\left(C_{\lambda}^{i}\right)=w\left(C_{\lambda}^{k}\right) \quad \text { for all } i, k .
$$

More precisely, we have

$$
C_{\lambda}^{i}=\cup_{k} \lambda_{k}^{i} \quad C_{\delta}^{i}=\cup_{k} \delta_{k}^{i}
$$

for a maximal chain $\left\{\lambda_{1}^{i}, \cdots, \lambda_{\ell}^{i}\right\}$ chosen 4.2 (Reduction). Since both $C_{\lambda}^{i}$ and $C_{\delta}^{i}$ are arcs (and so contractible to points) with the same images under $w$ and by the way how the maximal chain is defined, we can continuously deform $w$ into $\check{w}:(\widetilde{E}, \partial \widetilde{E}) \rightarrow(M, R)$ so that

$$
\check{w}\left(\cup C^{i}\right)=\{\text { one point }\}
$$

and

$$
[w]=[\check{w}] \quad \text { in } H_{2}(M, R ; \mathbb{Z}) .
$$

Obviously we also have

$$
\left[\left.\check{w}\right|_{\widetilde{E}^{i}}\right]=\left[\left.w\right|_{\widetilde{E}^{i}}\right] \quad \text { in } H_{2}(M, R ; \mathbb{Z}) .
$$

Now it is immediate to check that

$$
\left[\left.\check{w}\right|_{\widetilde{E}^{i}}\right]=\left[w^{i}\right] \quad \text { in } H_{2}(M, R ; \mathbb{Z}) .
$$

Hence the proof of Proposition A.4. 


\section{References.}

[BG] E. Bedford and B. Gaveau, Envelopes of holomorphy of certain 2-spheres in $\mathbb{C}^{2}$, Amer. J. Math. 105 (1983), 975-1009.

[C] E. M. Chirka, Regularities of the boundaries of analytic sets, Math. USSR Sbornik, 45 (1983), 291-335.

[F] A. Floer, Symplectic fixed points and holomorphic spheres, Commun. Math. Phys. 120 (1989), 575-611.

[FHS] A. Floer, H. Hofer, and D. Salamon, Transversality in elliptic Morse theory for symplectic actions, Duke Math. J. 80 (1995), 251-292.

[GS] J. Globevnik and E. Stout, Analytic discs with rectifiable simple closed curves as ends, Ann. Math. 129 (1988), 380-401.

[H] R. Hind, Filling by holomorphic discs with weakly pseudo-convex boundary conditions, Geom. Funct. Anal. 7 (1997), 462-495.

[HS] H. Hofer and D. Salamon, Floer homology and Novikov rings, in Floer Memorial Volume, Birkhauser, Basel, 1995; ed. Hofer, Taubes, Weinstein and Zehnder, 483-524.

[L] F. Lalonde, Hamiltonian collapsing of irrational Lagrangian submanifolds with small first Betti-number, Commun. Math. Phys. 149 (1992), 613-622.

[La] L. Lazzarini, Existence of a somewhere injective pseudo-holomorphic disc, preprint, 1998.

[M1] D. McDuff, Examples of symplectic structures, Invent. Math. 89 (1987), 13-30.

[M2] D. McDuff, The structure of rational and ruled symplectic 4-manifolds, J. Amer. Math. Soc. 3 (1990), 679-712. Erratum: ibid, 5 (1992), 987-988.

[M3] D. McDuff, The local behavior of holomorphic curves in almost complex 4-manifolds, J. Diff. Geom. 34 (1991), 143-164.

[M4] D. McDuff, Singularities of J-holomorphic curves in almost complex 4manifolds, J. Geom. Anal. 2 (1992), 249-266.

[MS] D. McDuff and D. Salamon, J-holomorphic Curves and Quantum Cohomology, Univ. Lec. Series 6, AMS, 1994.

[O1] Y.-G. Oh, Removal of boundary singularities of pseudo-holomorphic curves with Lagrangian boundary conditions, Comm. Pure Appl. Math. 45 (1992), 121-139. 
[O2] Y.-G. Oh, Floer cohomology of Lagrangian intersections and pseudoholomorphic discs, I, Comm. Pure Appl. Math. 46 (1993), 949-994. Addendum: ibid, 48 (1995), 1299-1302.

[O3] Y.-G. Oh, Fredholm theory of holomorphic discs under the perturbation of boundary conditions, Math. Z. 222 (1996), 505-520.

[O4] Y.-G. Oh On the structure of pseudo-holomorphic discs with totally real boundary conditions, J. Geom. Anal., 7 (1997), 305-327. Erratum; ibid, to appear.

[P] L. Polterovich, The Maslov class of Lagrange surface and Gromov's pseudoholomorphic curves, Trans. A.M.S. 325 (1992), 241-248.

[R] Y. Ruan, Topological sigma model and Donaldson type invariants in Gromov theory, Duke Math. J. 83 (1996), 461-500.

[RT] Y. Ruan and G. Tian, A mathematical theory of quantum cohomology, J. Diff. Geom. 42 (1995), 259-367.

[Si] J. C. Sikorav, Singularities of J-holomorphic curves, Math. Z. 226 (1997), $359-373$.

[St] E. Stout, Bounded extensions: The case of discs in polydiscs, J. Analyse Math. 10 (1975), 239-254.

[Y1] R. Ye, Gromov's compactness theorem for pseudo-holomorphic curves, Trans. A.M.S. 342 (1994), 671-694.

[Y2] R. Ye, Filling by holomorphic discs in symplectic 4-manifolds, Trans. A.M.S. 350 (1998), 213-250.

RECEIVEd APRIL 30, 1997.

SEoul National University,

SEOUL 151-742, KOREA

CURrent Address: Korea Institute for Advanced Study, 207-43 ChUNGRYANGRI-DONG DONGDAEMUN-GU

SEOUL 130-012, KOREA

AND

Korea Institute for Advanced Study, 207-43 CHUNGRYANGRI-DONG DONGDAEMUN-GU

SEOUL 130-012, KOREA \& 
UNIVERSITY OF WISCONSIN, MADISON, WI 53706, USA 